\title{
Synthesis of aryl-substituted indanones and indenes via a highly efficient ligand-free palladium-catalyzed Suzuki coupling process
}

\author{
Yuming Song, ${ }^{\text {a* }}$ Yamei Xie, ${ }^{\text {a }}$ Minjian Chen, ${ }^{\text {a Jingping Qu, }{ }^{a} \text { Hongfan Hu, }{ }^{b} \text { Xiaomei Lang, }}$ \\ Shan Xue, ${ }^{\mathrm{b}}$ Yin Ran, ${ }^{\mathrm{b}}$ and Shixuan Xin ${ }^{\mathrm{b} *}$ \\ a State Key Laboratory of Fine Chemicals, School of Pharmaceutical Science and Technology, \\ Dalian University of Technology. Dalian 116024, P. R. China \\ ${ }^{b}$ PetroChina Petrochemical Research Institute, Block A42, PetroChina Innovation Base, \\ Shahe Township, Changping District, Beijing 102206, P. R. China \\ E-mail:song-ym@dlut.edu.cn; xinshixuan@petrochina.com.cn
}

DOI: http://dx.doi.org/10.3998/ark.5550190.p009.627

\begin{abstract}
Strategically substituted indene derivatives are useful building blocks for high efficiency olefin polymerization metallocene catalysts. In this paper, various 4-aryl-substituted 2-methyl- $1 \mathrm{H}$ indanones were prepared efficiently using a ligand-free palladium-catalysed Suzuki coupling procedure. Quantitative yields of indanone intermediates were achieved for most of the noncoordinative substrates with a loading of $0.005 \mathrm{~mol} \%$ of palladium catalyst. The corresponding indene derivatives were obtained in high purity and multi-gram scale in excellent yields, following a simple sequential reduction and dehydration procedure.
\end{abstract}

Keywords: Suzuki coupling, ligand-free palladium catalysis, metallocenes, polymerization

\section{Introduction}

Among numerous highly active homogeneous olefin polymerization catalysts, racemic dimethylsilyl-bridged bis-2-methyl-4-phenylindenyl $\mathrm{ZrCl}_{2}$, (Figure1) reported by the Hoechst team in the early 1990s, acts as a cornerstone in isospecific propylene polymerization catalysis, producing isotactic polypropylene (iPP) with significantly high catalytic activity, high molecular mass, high iso-specificity, and high melting point for industrial applications. Known results have shown that methyl and phenyl substitutions are responsible for superior performance of the catalysts in respect of high molecular weight, isotacticity and activity. ${ }^{1-3}$ Subsequent research found that the precatalyst $\mathbf{1}$ is also a versatile catalyst for the preparation of olefin copolymers ${ }^{4-8}$ or heteroatom containing functionalized copolymers, ${ }^{9-14}$ which could potentially be used for the substitution of 
PS, PVC, polydiene and related copolymers or as specialty materials (for example coatings, blends, composites, or ion exchange membranes, etc.). Other metallocenes based on $\mathbf{1}$ have appeared since 1990, and intensive studies of substitution effects for all possible positions of the indene framework were carried out. Much better catalytic performances were achieved and the polymers or co-polymer materials so generated exhibit pronounced improvements of PP properties (melting temperature, molecular mass, uniform monomer distribution, comonomer incorporation, et al.). . $, 3,15-18^{-1}$

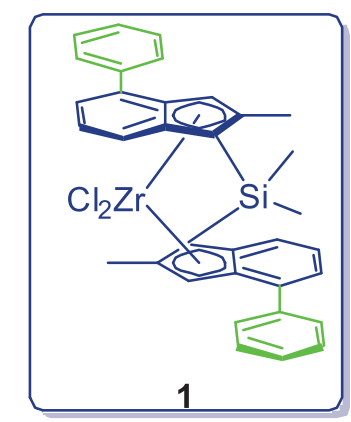

Figure 1. The Hoechst catalyst.

The critical dependence of the activity and selectivity of a metallocene on its ligand structure, especially the fact that some of the $C_{1}$ symmetric analogues exhibit boosted catalytic performances compared with their $C_{2}$ symmetric counterparts, have stimulated continuing efforts to develop fast and reliable ligand synthetic processes. Many research groups have developed diverse coupling procedures for the synthesis of substituted indenes, based on abundant starting materials and highly efficient organic transformations. There are several strategies applied according to different coupling precursors:

A) From the coupling of 2-functionalized toluenes with desired aryl partners, a reliable sequential procedure was established and a number of indene ligands were prepared. However, many repeated operations are needed to evaluate catalysts with various aryl substitutions. In addition, undesired side reactions may occur for some sensitive substrates during the tedious process. For example, an extremely low yield was observed during the preparation of 2-methyl-4-(1-naphthyl)indanone using the above mentioned procedure; ${ }^{1,19}$

B) As an important improvement, phosphine- or nitrogen-containing palladium complexes catalyzed Suzuki-Miyaura coupling of 4/7-halo indanones was widely applied in 4/7-aryl indene syntheses. Nevertheless, extra ligands used in these reactions caused additional limits such as inert atmosphere protection, more complex purification procedures or higher catalyst loadings. ${ }^{7,18,20,21}$ C) Alexander et al. reported an impressive procedure of catalyzed coupling of 4/7 functionalized indenes or indanes with arylboron, halogen, zinc, or magnesium reagents, using metal complexes, as the key step, affording 4-/7-aryl-substituted indenes in excellent yields. This method has been 
widely used in novel indene ligand synthesis. However, besides the above-mentioned limits with method $\mathbf{B}$, the use of organometallic reagents greatly narrows the substrate scope. ${ }^{22-24}$

D) As the most straightforward strategy, bromo-substituted Group 4 metallocenes can be efficiently coupled with organo-zinc reagents following a Negishi coupling procedure. However, strikingly lower isolated yields were obtained for some of the substrates because of their sensitivity or isolation problems (Scheme 1). ${ }^{25-27}$ Most of these methods need extra ligands to stabilize the palladium catalysts, relatively high noble metal catalyst loading (2-6 mol\%) and some of the organometallic reagents utilized severely limit the reaction conditions and the substrate scope.

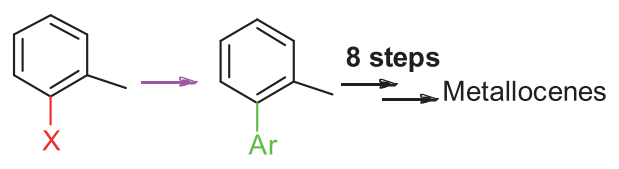

Suzuki-Miyaura, Kumada, Negishi

A

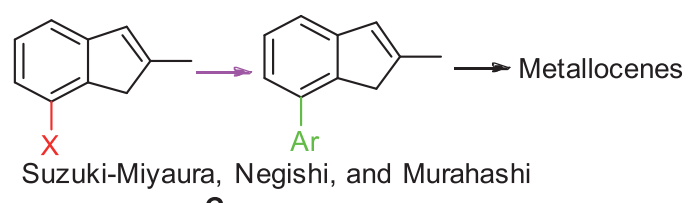

C

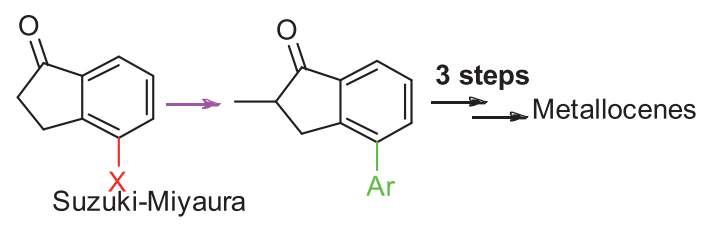

B

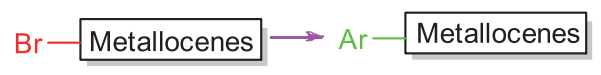

D

Scheme 1. General methods for Ar-Ar bond formation in metallocene synthesis.

In recent years, many computational studies have been reported aimed at understanding olefin polymerization mechanisms, which have stimulated the development of novel metallocenes useful for new plastic material production. ${ }^{28-31}$ In our continuing efforts to develop highly efficient metallocene catalysts based on theoretical computation and high-throughput methods for specialty polyolefins, a simple and efficient synthesis of high purity 4/7-arylindene derivatives is, undoubtedly, of great importance. In this respect, special attention has been paid to 4-arylindanones 4, which can easily be converted into substituted 7-arylindenes $\mathbf{5}$ and thence into electron-rich ligands 6 following known procedures (Scheme 2). Moreover, the brominated indanones $\mathbf{2}$ could easily be prepared from abundant commercially available materials. Also, the electron-withdrawing property of the ketone group facilitates the oxidative addition of aryl bromide to the palladium center, which in most cases is known as the rate determining step in the catalytic cycle (Scheme 3). ${ }^{32-35}$ PEG-mediated ligand-free Suzuki coupling reactions are attractive because they avoid the use of a complex ligand, thus reducing the residue of harmful and costly noble metals in the final product and simplifying work-up procedures. ${ }^{36-39}$

We report here a highly efficient ligand-free catalytic system for the Suzuki coupling of 4-bromo-2-methyl-1 $H$-indanone with aryl/heteroaryl boronic acids in a tetrabutylammonium bromide (TBAB)/Pd(OAc) 2 / PEG400 system. Most of the reactions were complete in one hour at $110{ }^{\circ} \mathrm{C}$ without inert gas protection. Following a prototype reduction and dehydration procedure, the final 7-aryl-2-methyl- $1 H$-indene products could be prepared in excellent yields. Multi-gram 
scale reactions of 4-bromo-2-methyl- $1 H$-indanone with 3,5-bis(trifluoromethyl)phenylboronic acid as the substrate proceeded smoothly, and the substituted indene was prepared in very high total yield for three steps without fractional distillation or column chromatography.

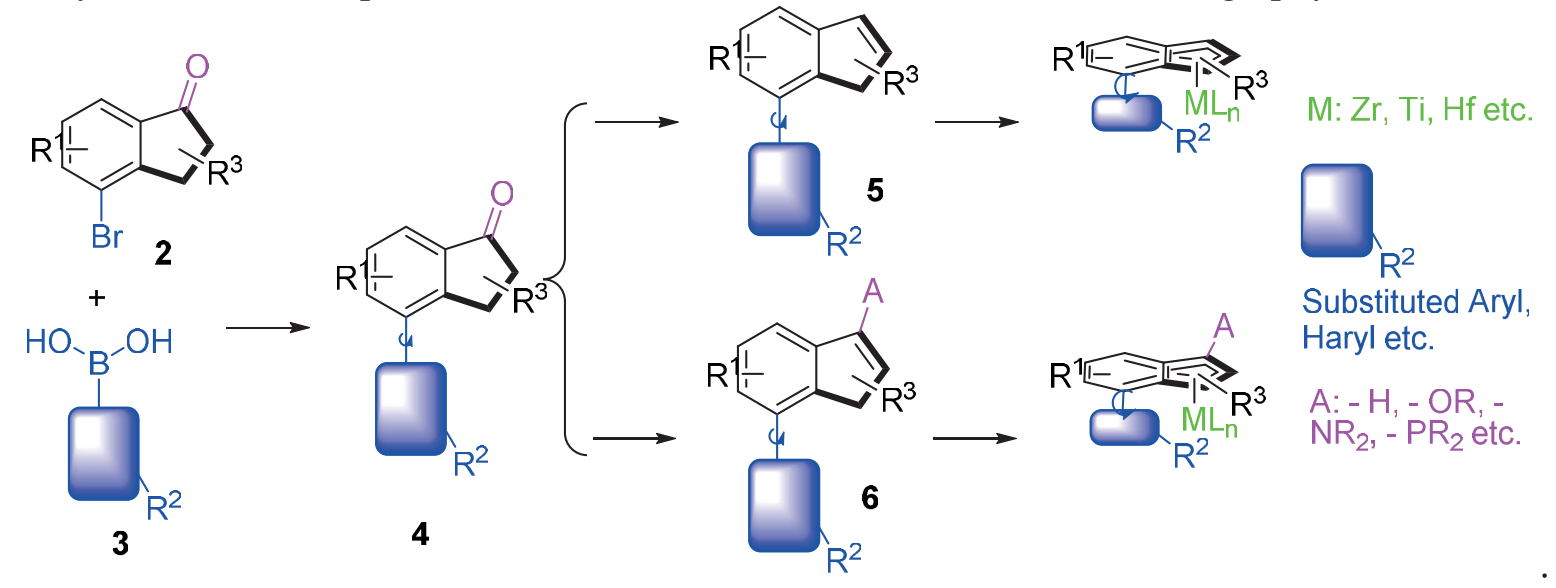

Scheme 2. Metallocenes prepared from 4-bromoindan-1-one.

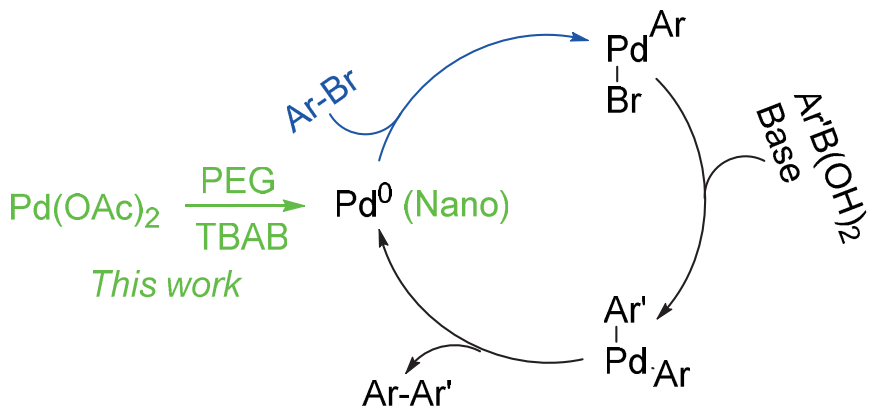

Scheme 3. General catalytic cycle of the Suzuki-Miyaura coupling.

\section{Results and Discussion}

The Suzuki coupling of 4-bromo-2-methylindan-1-one with phenylboronic acid (8a) in a $\mathrm{PEG} 400 / \mathrm{Pd}(\mathrm{OAc})_{2} / \mathrm{TBAB}$ system was chosen as the model reaction and various parameters were evaluated (Equation 1). The reaction yield improved from $17 \%$ to $98 \%$ in one hour at elevated temperatures (from $80^{\circ} \mathrm{C}$ to $110{ }^{\circ} \mathrm{C}$ ). In situ generated nano palladium particles, whose surface properties are unambiguously affected by reaction temperature, have been proved to be active catalysts (Table 1 entries 1,2$).{ }^{34}$ Of those tested, potassium carbonate was the base of choice, providing the highest product yields (Table 1, entries 2-5), while potassium hydroxide was a poor base for the coupling (Table 1, entry 3). Sodium carbonate and potassium phosphate also gave good yields (Table 1, entries 4, 5). In addition, the effect of TBAB on the reaction was examined under otherwise identical conditions; reactions without $\mathrm{TBAB}$ or decreasing its loading to 10 
mol\% furnished the coupled product in lower yields. Reported results showed that TBAB played a dual role for the reaction, as phase transfer catalyst and also as a nano-palladium stabilizer (Table 1, entries 6, 7). Surprisingly, on reducing the loading of the noble metal catalyst precursor $\mathrm{Pd}(\mathrm{OAc})_{2}$ from $0.1 \mathrm{~mol} \%$ to $0.01 \mathrm{~mol} \%$, or even to as low as $0.005 \mathrm{~mol} \%$, identical catalytic productivities were achieved under otherwise identical reaction conditions. Further decreasing the catalyst loading to $0.001 \mathrm{~mol} \%$ resulted in a lowered yield of coupling product $53 \%$ in three hours). To the best of our knowledge, this is one of the most efficient methods for this kind of indanone synthesis to date (Table 1 , entries $8,9,10$ ). As a comparison, the same coupling was performed under the commonly used oxygen-free coupling conditions with $0.1 \mathrm{~mol} \%$ of $\mathrm{Pd}\left(\mathrm{PPh}_{3}\right)_{4}$ as the catalyst, and $90 \%$ of coupling yield was obtained in five hours (Table 1, entry 11).

Table 1. Suzuki coupling of 4-bromo-2,3-dihydro-2-methyl-1 $H$-inden-1-one 7 with phenylboronic acid $8 \mathbf{a}_{0}{ }^{\text {a }}$

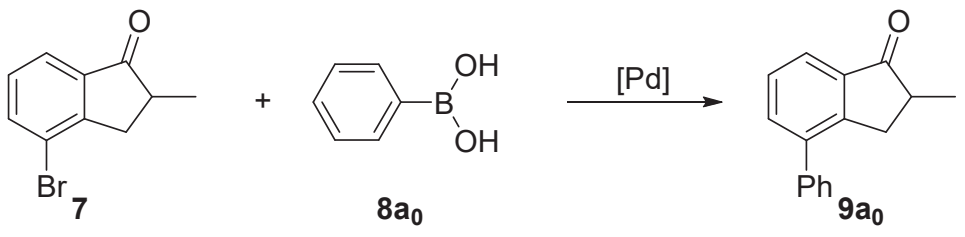

(1)

\begin{tabular}{ccccccc}
\hline Entries & Catalyst & Base & TBAB & Temp $\left({ }^{\circ} \mathrm{C}\right)$ & Time $(\mathrm{h})$ & ${\text { Yield }(\%)^{\mathrm{b}}}^{\mathrm{n}}$ \\
\hline 1 & $\mathrm{Pd}(\mathrm{OAc})_{2}$ & $\mathrm{~K}_{2} \mathrm{CO}_{3}$ & 1 eq. & 80 & 1 & 17 \\
2 & $\mathrm{Pd}(\mathrm{OAc})_{2}$ & $\mathrm{~K}_{2} \mathrm{CO}_{3}$ & 1 eq. & 110 & 1 & 98 \\
3 & $\mathrm{Pd}(\mathrm{OAc})_{2}$ & $\mathrm{KOH}$ & 1 eq. & 110 & 1 & 27 \\
4 & $\mathrm{Pd}(\mathrm{OAc})_{2}$ & $\mathrm{Na}_{2} \mathrm{CO}_{3}$ & 1 eq. & 110 & 1 & 74 \\
5 & $\mathrm{Pd}(\mathrm{OAc})_{2}$ & $\mathrm{~K}_{3} \mathrm{PO}_{4}$ & 1 eq. & 110 & 1 & 76 \\
6 & $\mathrm{Pd}(\mathrm{OAc})_{2}$ & $\mathrm{~K}_{2} \mathrm{CO}_{3}$ & none & 110 & 12 & 34 \\
7 & $\mathrm{Pd}(\mathrm{OAc})_{2}$ & $\mathrm{~K}_{2} \mathrm{CO}_{3}$ & 10 mol\% & 110 & 1 & 85 \\
$8^{\mathrm{c}}$ & $\mathrm{Pd}(\mathrm{OAc})_{2}$ & $\mathrm{~K}_{2} \mathrm{CO}_{3}$ & 1 eq. & 110 & 1 & 95 \\
$9^{\mathrm{d}}$ & $\mathrm{Pd}(\mathrm{OAc})_{2}$ & $\mathrm{~K}_{2} \mathrm{CO}_{3}$ & 1 eq. & 110 & 1 & 98 \\
$10^{\mathrm{e}}$ & $\mathrm{Pd}(\mathrm{OAc})_{2}$ & $\mathrm{~K}_{2} \mathrm{CO}_{3}$ & 1 eq. & 110 & 3 & 53 \\
$11^{\mathrm{f}}$ & $\mathrm{Pd}\left(\mathrm{PPh}_{3}\right)_{4}$ & $\mathrm{~K}_{2} \mathrm{CO}_{3}$ & none & 90 & 5 & 90 \\
\hline
\end{tabular}

a $7(0.2 \mathrm{mmol}), 8 \mathbf{a}_{0}(0.24 \mathrm{mmol})$, base, $\mathrm{Pd}(\mathrm{OAc})_{2}(0.1 \mathrm{~mol} \%)$, PEG400 $(1 \mathrm{~g}) ;{ }^{\mathrm{b}} \mathrm{GC}$-area normalization;

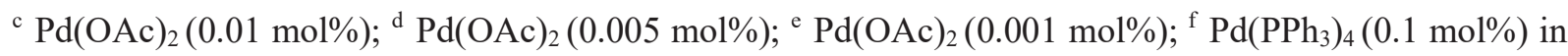
EtOH- $\mathrm{H}_{2} \mathrm{O}$.

With the process established, a variety of coupling reactions of 4-bromo-2-methylindan-1one with substituted phenylboronic acids were investigated (Table 2, Equation 2). 
Table 2. The Suzuki coupling of 4-bromo-2,3-dihydro-2-methyl-1H-inden-1-one 7 and arylboronic acids $\mathbf{8}^{\text {a }}$

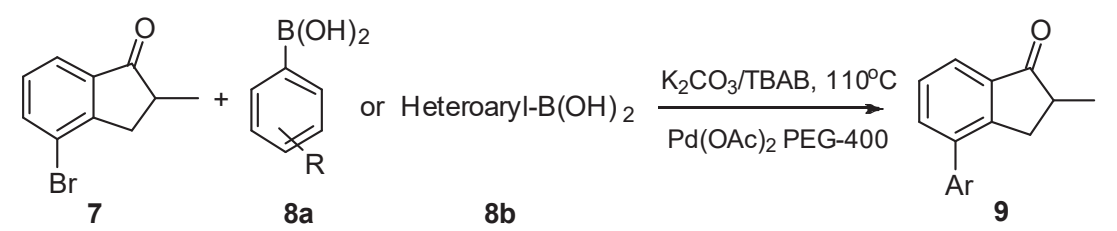

\begin{tabular}{|c|c|c|c|c|c|c|}
\hline Entry & $\begin{array}{c}\text { Boronic acid } \\
\mathbf{8} \\
\end{array}$ & $\begin{array}{c}\mathrm{R} \text { or } \\
(\text { Heteroaryl }=\mathrm{Ar})\end{array}$ & Cat. (mol\%) & $\begin{array}{l}\text { Time } \\
(\mathrm{h})\end{array}$ & $\begin{array}{c}\text { Product } \\
9\end{array}$ & $\begin{array}{c}\text { Isolated } \\
\text { yield (\%) }\end{array}$ \\
\hline 1 & $\mathbf{8} \mathbf{a}_{1}$ & $\mathrm{R}=2-\mathrm{CH}_{3}$ & 0.005 & 1 & $9 \mathbf{a}_{1}$ & 84 \\
\hline 2 & $8 \mathbf{a}_{2}$ & $\mathrm{R}=3-\mathrm{CH}_{3}$ & 0.005 & 1 & $9 \mathbf{a}_{2}$ & 90 \\
\hline 3 & $8 a_{3}$ & $\mathrm{R}=2-\mathrm{OCH}_{3}$ & 0.005 & 1 & $9 a_{3}$ & 89 \\
\hline 4 & $8 \mathbf{a}_{4}$ & $\mathrm{R}=3-\mathrm{OCH}_{3}$ & 0.005 & 1 & $9 \mathbf{a}_{4}$ & 94 \\
\hline 5 & $8 a_{5}$ & $\mathrm{R}=4-\mathrm{OCH}_{3}$ & 0.005 & 1 & $9 a_{5}$ & 91 \\
\hline 6 & $8 \mathbf{a}_{6}$ & $\mathrm{R}=3,5-\mathrm{di}-\mathrm{CH}_{3}$ & 0.005 & 1 & $9 a_{6}$ & 98 \\
\hline 7 & $8 \mathbf{a}_{7}$ & $\mathrm{R}=4-\mathrm{C}\left(\mathrm{CH}_{3}\right)_{3}$ & 0.005 & 1 & $9 \mathbf{a}_{7}$ & 94 \\
\hline 8 & $8 \mathbf{a}_{8}$ & $\mathrm{R}=4-\mathrm{OCF}_{3}$ & 0.005 & 1 & $9 a_{8}$ & 90 \\
\hline 9 & $8 a_{9}$ & $\mathrm{R}=2-\mathrm{F}$ & 0.005 & 3 & $9 a_{9}$ & 85 \\
\hline 10 & $8 a_{10}$ & $\mathrm{R}=3-\mathrm{F}$ & 0.005 & 1 & $9 a_{10}$ & 95 \\
\hline 11 & $8 a_{11}$ & $\mathrm{R}=4-\mathrm{F}$ & 0.005 & 1 & $9 \mathrm{a}_{11}$ & 97 \\
\hline 12 & $8 a_{12}$ & $\mathrm{R}=4-\mathrm{Cl}$ & 0.005 & 0.5 & $9 a_{12}$ & 90 \\
\hline 13 & $8 \mathbf{a}_{13}$ & $\mathrm{R}=3-\mathrm{CF}_{3}$ & 0.005 & 1 & $9 \mathrm{a}_{13}$ & 87 \\
\hline 14 & $8 a_{14}$ & $\mathrm{R}=4-\mathrm{CF}_{3}$ & 0.005 & 1 & $9 \mathbf{a}_{14}$ & 90 \\
\hline 15 & $8 \mathbf{a}_{15}$ & $\mathrm{R}=3,5-\mathrm{di}-\mathrm{CF}_{3}$ & 0.005 & 1 & $9 a_{15}$ & 98 \\
\hline 16 & $8 \mathbf{a}_{16}$ & $\mathrm{R}=3-\mathrm{CN}$ & 0.005 & 1 & $9 a_{16}$ & 90 \\
\hline 17 & $8 \mathbf{a}_{17}$ & $\mathrm{R}=4-\mathrm{CN}$ & 0.005 & 1 & $9 a_{17}$ & 96 \\
\hline 18 & $8 a_{18}$ & $\mathrm{R}=4-\mathrm{Ph}$ & 0.005 & 1 & $9 a_{18}$ & 82 \\
\hline 19 & $8 a_{19}$ & $\mathrm{R}=2-\mathrm{Cl}$ & 0.01 & 2 & $9 a_{19}$ & 90 \\
\hline 20 & $8 \mathbf{a}_{20}$ & $\mathrm{R}=3-\mathrm{Cl}$ & 0.01 & 0.5 & $9 \mathrm{a}_{20}$ & 87 \\
\hline 21 & $8 a_{21}$ & $\mathrm{R}=3-\mathrm{NO}_{2}$ & 0.01 & 1 & $9 a_{21}$ & 80 \\
\hline 22 & $8 \mathbf{a}_{22}$ & $\mathrm{R}=2-\mathrm{CF}_{3}$ & 5 & 1 & $9 a_{22}$ & 79 \\
\hline 23 & $8 \mathbf{a}_{23}$ & $\mathrm{Ar}=2$-naphthyl & 1 & 1 & $9 \mathrm{a}_{23}$ & 85 \\
\hline 24 & $8 b_{1}$ & (3-pyridinyl) & 1 & 1 & $9 b_{1}$ & 73 \\
\hline 25 & $8 b_{2}$ & (4-pyridinyl) & 1 & 1 & $9 b_{2}$ & 84 \\
\hline 26 & $8 b_{3}$ & (5-pyrimidinyl) & 1 & 1 & $9 b_{3}$ & 71 \\
\hline 27 & $8 b_{4}$ & (3-quinolinyl) & 1 & 1 & $9 b_{4}$ & 59 \\
\hline 28 & $8 b_{5}$ & (2-thienyl) & 5 & 1 & $9 b_{5}$ & 68 \\
\hline 29 & $8 b_{6}$ & (2-furyl) & 0.01 & 0.5 & $9 b_{6}$ & 90 \\
\hline
\end{tabular}

a 7 (0.2 mmol), 8 (0.24 mmol), $\mathrm{K}_{2} \mathrm{CO}_{3}(0.4 \mathrm{mmol}), \mathrm{Pd}(\mathrm{OAc})_{2}, \mathrm{PEG} 400(1 \mathrm{~g}), 110^{\circ} \mathrm{C}$ 
To our satisfaction, all the coupling reactions proceeded smoothly with substituted phenylboronic acids containing either electron-withdrawing or -donating groups $\left(\mathrm{CN}, \mathrm{CF}_{3}, t-\mathrm{Bu}, \mathrm{OMe}\right.$, et al.) with $0.005 \mathrm{~mol} \%$ of catalyst loading in $84-97 \%$ isolated yields (Table 2, entries $1-18$ ). For 2-Cl, $3-\mathrm{Cl}$ or $3-\mathrm{NO}_{2}$ phenylboronic acid, slightly elevated $\mathrm{Pd}(\mathrm{OAc})_{2}$ loading $(0.01 \mathrm{~mol} \%)$ is necessary for satisfactory coupling yields (80-90\%, Table 2, entries 19-21). Generally, the ortho substituted phenylboronic acids produced a somewhat inferior result to their meta and para substituted congeners, thus the reaction of 2-trifluoromethylphenylboronic acid needed as high as $5 \mathrm{~mol} \%$ of catalyst loading to deliver sufficient catalytic productivity (79\% yield). We ascribed this to the stereo-hindrance effect of the substrates (Table 2 Entries 1,3,9,19,22). 2-Naphthaleneboronic acid and heteroaryl boronic acids proved to be good candidates for the current coupling with 1-5 mol\% of catalyst precursor (Table 2, entries 23 - 28). The furan ring had much less effect on the reactivity than did N-containing heterocycles; thus with $0.01 \mathrm{~mol} \%$ of catalyst loading, the reaction was complete within 30 minutes to give the desired product in $90 \%$ isolated yield (Table 2, entry 29).

7-Aryl-2-methyl-1H-indenes were prepared following a reduction/dehydration procedure (Equation 3) and the results are listed in Table 3.

Table 3. Preparation of 7-aryl-2-methyl-1H-indenes $\mathbf{1 0}$ from 4-aryl-2-methyl-1-indanones $\mathbf{9}^{\mathrm{a}, \mathrm{b}}$

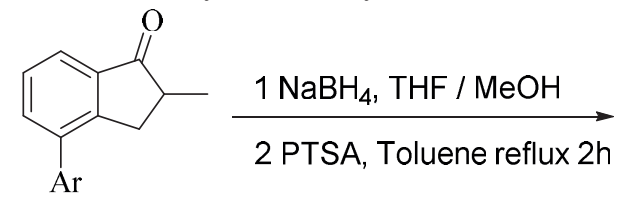

9

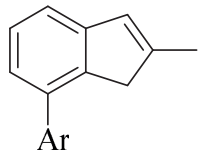

10
(3)

\begin{tabular}{|c|c|c|c|c|c|c|c|}
\hline Entry & Substrate & Product & Yield $^{\mathrm{c}}(\%)$ & Entry & Substrate & Product & Yield $^{\mathrm{c}}(\%)$ \\
\hline 1 & $9 a_{1}$ & $10 \mathbf{a}_{1}$ & 82 & 14 & $9 a_{14}$ & $10 a_{14}$ & 97 \\
\hline 2 & $9 \mathbf{a}_{2}$ & $10 a_{2}$ & 85 & 15 & $9 a_{15}$ & $10 a_{15}$ & 87 \\
\hline 3 & $9 a_{3}$ & $10 a_{3}$ & 79 & 16 & $9 a_{16}$ & $10 a_{16}$ & 93 \\
\hline 4 & $9 a_{4}$ & $10 a_{4}$ & 90 & 17 & $9 \mathbf{a}_{17}$ & $10 a_{17}$ & 75 \\
\hline 5 & $9 a_{5}$ & $10 a_{5}$ & 95 & 18 & $9 a_{18}$ & $10 a_{18}$ & 91 \\
\hline 6 & $9 a_{6}$ & $10 a_{6}$ & 93 & 19 & $9 a_{20}$ & $10 \mathbf{a}_{20}$ & 89 \\
\hline 7 & $9 a_{7}$ & $10 a_{7}$ & 94 & 20 & $9 a_{23}$ & $10 \mathbf{a}_{23}$ & 94 \\
\hline 8 & $9 \mathrm{a}_{8}$ & $10 a_{8}$ & 96 & 21 & $9 b_{1}$ & $10 b_{1}$ & 89 \\
\hline 9 & $9 a_{9}$ & 10a9 & 97 & 22 & $9 b_{2}$ & $10 b_{2}$ & 98 \\
\hline 10 & $9 \mathbf{a}_{10}$ & $10 a_{10}$ & 96 & 23 & $9 b_{3}$ & $10 b_{3}$ & 79 \\
\hline 11 & $9 a_{11}$ & $10 a_{11}$ & 90 & 24 & $9 b_{4}$ & $10 b_{4}$ & 53 \\
\hline 12 & $9 \mathbf{a}_{12}$ & $10 a_{12}$ & 77 & 25 & $9 b_{5}$ & $10 b_{5}$ & 77 \\
\hline 13 & $9 \mathbf{a}_{13}$ & $10 a_{13}$ & 90 & 26 & $9 b_{6}$ & $10 b_{6}$ & 63 \\
\hline
\end{tabular}

aReduction: 9 (1.0 mmol), $\mathrm{NaBH}_{4}(3.0 \mathrm{mmol}), \mathrm{THF} / \mathrm{MeOH}(15 \mathrm{~mL} 2: 1), 0{ }^{\circ} \mathrm{C} \sim \mathrm{r} . \mathrm{t}, 4 \mathrm{~h}$. bDehydration: PTSA (100 mg), toluene $(50 \mathrm{~mL})$, reflux, $2 \mathrm{~h} .{ }^{\mathrm{c}}$ Isolated yield. 
Most of the reactions proceeded smoothly and produced the desired indene products in excellent yields. It is should be noted that higher concentrations in the dehydration step may promote undesirable side reactions, especially for some electron-rich substrates.

Considering the solubility differences of PEG400, $\mathrm{Pd}(\mathrm{OAc})_{2}, \mathrm{TBAB}$, arylboronic acids, substituted indanones and related indenes, developing a fast and highly efficient 7-aryl-2-alkyl$1 H$-indene synthetic procedure with simple purification operations is highly desirable. To this end, 3,5-bis(trifluoromethyl)phenylboronic acid was chosen as the model substrate for a multi-gram scale (4.5 g, $20 \mathrm{mmol}$ ) synthesis following the current procedure. According to previous results, the couplings were fast, clean and previously observed side reactions, such as debromination and/or de-boronation were, to our surprise, not observed; thus the aryl boronic acid was used in slight excess (1.05 eq.). As expected, the reaction proceeded smoothly at higher concentration and was accomplished with only $0.005 \mathrm{~mol} \%$ of catalyst loading. After normal extraction and evaporation, complete removal of residual PEG400, $\mathrm{Pd}(\mathrm{OAc})_{2}$, TBAB and aryl boronic acid was achieved by washing with cold methanol. After the reduction and dehydration protocol, the crude product was washed again with methanol to give $86 \%$ of pure indene derivative $\mathbf{1 0 a _ { 1 5 }}$ as a white crystalline material (Scheme 4).<smiles>CC1Cc2c(Br)cccc2C1=O</smiles>

7

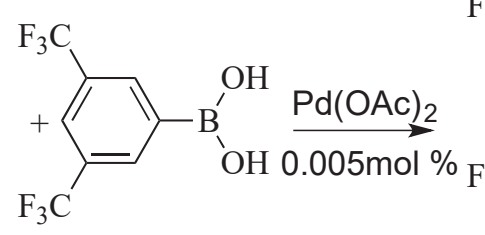

$8 a_{15}$

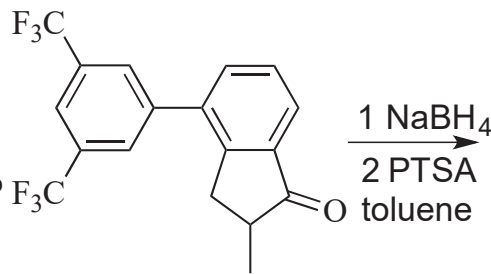

$9 a_{15}$

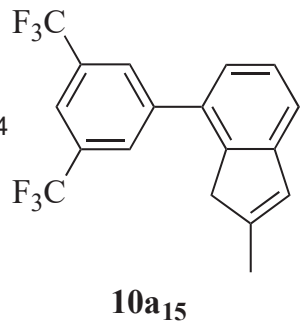

$5.9 \mathrm{~g}(17 \mathrm{mmol}) 86 \%$

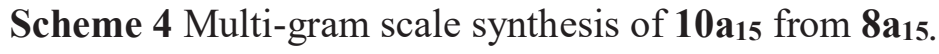

\section{Conclusions}

A ligand-free Suzuki coupling system consisting of PEG-400/Pd(OAc) $)_{2} / \mathrm{TBAB} / \mathrm{K}_{2} \mathrm{CO}_{3}$ in optimized ratio was employed for the Suzuki-Miyaura coupling reaction of 4-bromo-2,3-dihydro2-methyl-1H-inden-1-one (7) with aryl and/or heteroarylboronic acids. Most of the substituted phenylboronic acids reacted smoothly with $0.005 \mathrm{~mol} \%$ of catalyst loading, and all the reactions were accomplished within a period of 0.5-3 hours in excellent yields (82-98\%). Some of the heteroarylboronic acids also reacted in good to excellent yield (59-90\%) with controlled low catalyst loading $(0.01-5 \mathrm{~mol} \%)$. The intermediate indanones could be easily transformed into their indene derivatives in high purity and high productivity. Multi-gram scale reaction of $\mathbf{8} \mathbf{a}_{15}$ was conducted following our typical Suzuki-Miyaura coupling, reducing and dehydrating procedures without fractional distillation or column chromatographic purification. Pure substituted indene 10a15 was obtained in high yield. Coupling of more complex substituted indanones with 
arylboronic acids following the current procedure is in progress and the catalytic properties of newly prepared novel $\mathrm{C} 1$ and $\mathrm{C} 2$ symmetric metallocenes are under evaluation.

\section{Experimental Section}

General. Melting points were measured on a Novel X-5 melting point instrument. All ${ }^{1} \mathrm{H}$ NMR $(400 \mathrm{MHz})$ and ${ }^{13} \mathrm{C}$ NMR $(100 \mathrm{~Hz})$ spectra were measured in $\mathrm{CDCl}_{3}$ and recorded on Bruker Avance II $400\left({ }^{1} \mathrm{H}\right.$ NMR) spectrometer with chemical shifts reported as ppm (with TMS as an internal standard). Purification of the reaction products was carried out by flash chromatography (FC) on silica gel (200-300 mesh). HRMS were conducted on GCT mass spectrometer (EI). All reactions were carried out in air and using distilled solvents, without any precautions to exclude moisture unless otherwise noted. Commercial grade reagents and solvents were used without further purification; otherwise, where necessary, they were purified as recommended.

4-Bromo-2,3-dihydro-2-methyl-1H-inden-1-one 7 was prepared from 2-bromobenzyl bromide following a reported ${ }^{22}$ procedure in $85 \%$ yield. $\mathrm{Mp} 40-42{ }^{\circ} \mathrm{C} .{ }^{1} \mathrm{H} \mathrm{NMR}\left(\mathrm{CDCl}_{3}, 400 \mathrm{MHz}\right) \delta_{\mathrm{H}}: 7.74$ $\left(1 \mathrm{H}, \mathrm{d},{ }^{3} J_{\mathrm{HH}} 7.8 \mathrm{~Hz}, \mathrm{ArH}\right), 7.69\left(1 \mathrm{H}, \mathrm{d},{ }^{3} J_{\mathrm{HH}} 7.5 \mathrm{~Hz}, \mathrm{ArH}\right), 7.27\left(1 \mathrm{H}, \mathrm{t},{ }^{3} J_{\mathrm{HH}} 7.7 \mathrm{~Hz}, \mathrm{ArH}\right), 3.31-$ $3.38\left(1 \mathrm{H}, \mathrm{dd},{ }^{3} J_{\mathrm{HH}} 7.6 \mathrm{~Hz},{ }^{2} J_{\mathrm{HH}} 17.6 \mathrm{~Hz}, \mathrm{CH}_{2} \mathrm{CH}\right), 2.71-2.81\left(\mathrm{H}, \mathrm{m}, \mathrm{CH}_{2} \mathrm{CH}\right), 2.67\left(1 \mathrm{H}, \mathrm{dd},{ }^{3} J_{\mathrm{HH}}\right.$ $\left.4.0 \mathrm{~Hz},{ }^{2} \mathrm{~J}_{\mathrm{HH}} 17.6 \mathrm{~Hz}, \mathrm{CH}_{2} \mathrm{CH}\right), 1.32\left(3 \mathrm{H}, \mathrm{d},{ }^{3} \mathrm{~J}_{\mathrm{HH}} 7.4 \mathrm{~Hz}, \mathrm{CHCH}_{3}\right)$.

\section{General procedure for the Suzuki coupling reaction}

Into a $10 \mathrm{~mL}$ vial, was filled with a mixture of 4-bromo-2,3-dihydro-2-methyl-1H-inden-1-one 7 (44.8 mg, $0.20 \mathrm{mmol}, \mathrm{PhB}(\mathrm{OH})_{2} \mathbf{8 \mathbf { a } _ { 0 }}\left(26.8 \mathrm{mg}, 0.22 \mathrm{mmol}, 1.2\right.$ eq.), $\mathrm{Pd}(\mathrm{OAc})_{2}$ (chloroform solution, $0.005 \mathrm{~mol} \%$ ), TBAB (75.7 mg, $0.20 \mathrm{mmol}, 1.0$ eq.), $\mathrm{K}_{2} \mathrm{CO}_{3}$ (55.3 mg, $0.40 \mathrm{mmol}, 2.0$ equiv) and PEG400 1.0 g. The vial was capped and the mixture was stirred at $110^{\circ} \mathrm{C}$ till completion (TLC). $5 \mathrm{~mL}$ of water was added and the contents were extracted with EtOAc $(10 \mathrm{~mL} \times 3)$, the combined organic phases were washed with brine $(10 \mathrm{~mL} \times 3)$, dried over $\mathrm{MgSO}_{4}$ and concentrated. The residue was subjected to column chromatography to obtain the desired product 9a $\mathbf{a}_{0}$ in $98 \%$ yield. ${ }^{1} \mathrm{H} \mathrm{NMR}\left(\mathrm{CDCl}_{3}, 400 \mathrm{MHz}\right) \delta_{\mathrm{H}}: 7.78\left(1 \mathrm{H}, \mathrm{d},{ }^{3} J_{\mathrm{HH}} 8.4 \mathrm{~Hz}, \mathrm{ArH}\right), 7.61(1 \mathrm{H}, \mathrm{d}$, $\left.{ }^{3} J_{\mathrm{HH}} 7.4 \mathrm{~Hz}, \mathrm{ArH}\right), 7.45-7.50(5 \mathrm{H}, \mathrm{m}, \mathrm{ArH}), 7.40-7.43(1 \mathrm{H}, \mathrm{m}, \mathrm{ArH}), 3.40-3.46\left(1 \mathrm{H}, \mathrm{dd},{ }^{3} J_{\mathrm{HH}} 7.6\right.$ $\left.\mathrm{Hz},{ }^{2} \mathrm{JHH} 17.6 \mathrm{~Hz}, \mathrm{CH}_{2} \mathrm{CH}\right), 2.61-2.81\left(2 \mathrm{H}, \mathrm{m}, \mathrm{CH}_{2} \mathrm{CH}\right), 1.32\left(3 \mathrm{H}, \mathrm{d},{ }^{3} \mathrm{~J}_{\mathrm{HH}} 7.3 \mathrm{~Hz}, \mathrm{CHCH}_{3}\right)$.

2-Methyl-4-(o-tolyl)-2,3-dihydro-1H-inden-1-one (9a). Yield: 84\%, $198 \mathrm{mg}$, colorless oil. ${ }^{1} \mathrm{H}$ NMR $\left(\mathrm{CDCl}_{3}, 400 \mathrm{MHz}\right) \delta_{\mathrm{H}}: 7.75(1 \mathrm{H}, \mathrm{m}, \mathrm{ArH}), 7.41-7.43(2 \mathrm{H}, \mathrm{m}, \mathrm{ArH}), 7.28-7.30$ (2H, m, ArH), 7.22-7.27 (1H, m, ArH), $7.14\left(1 \mathrm{H}, \mathrm{d},{ }^{3} J_{\mathrm{HH}} 7.1 \mathrm{~Hz}, \mathrm{ArH}\right), 3.07\left(1 \mathrm{H}, \mathrm{br}, \mathrm{CH}_{2} \mathrm{CH}\right), 2.64-2.69(1 \mathrm{H}, \mathrm{m}$, $\left.\mathrm{CH}_{2} \mathrm{CH}\right), 2.44\left(1 \mathrm{H}, \mathrm{br}, \mathrm{CH}_{2} \mathrm{CH}\right), 2.11\left(3 \mathrm{H}, \mathrm{s}, \mathrm{ArCH}_{3}\right), 1.25\left(3 \mathrm{H}, \mathrm{d},{ }^{3} \mathrm{JHH}_{\mathrm{HH}} 7.4 \mathrm{~Hz}, \mathrm{CHCH}_{3}\right) .{ }^{13} \mathrm{C} \mathrm{NMR}$ $\left(\mathrm{CDCl}_{3}, 100 \mathrm{MHz}\right) \delta_{\mathrm{C}}: 209.4,151.7,140.2,138.5,136.4,135.4,134.9,130.2,128.9,127.8,127.4$, 125.7, 122.7, 41.9, 34.2, 19.2, 19.8, 16.1. EI-HRMS $(\mathrm{m} / \mathrm{z})$ calcd for $\mathrm{C}_{17} \mathrm{H}_{16} \mathrm{O}\left(\mathrm{M}^{+}\right) 236.1201$, found 236.1200 . 


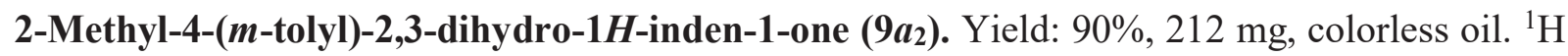
NMR $\left(\mathrm{CDCl}_{3}, 400 \mathrm{MHz}\right) \delta_{\mathrm{H}}: 7.81\left(1 \mathrm{H}, \mathrm{d},{ }^{3} J_{\mathrm{HH}} 7.6 \mathrm{~Hz}, \mathrm{ArH}\right), 7.61-7.65(1 \mathrm{H}, \mathrm{m}, \mathrm{ArH}), 7.50(1 \mathrm{H}$, t, $\left.{ }^{3} J_{\mathrm{HH}} 7.5 \mathrm{~Hz}, \mathrm{ArH}\right), 7.41\left(1 \mathrm{H}, \mathrm{t},{ }^{3} J_{\mathrm{HH}} 7.6 \mathrm{~Hz}, \operatorname{ArH}\right), 7.32(1 \mathrm{H}, \mathrm{s}, \operatorname{ArH}), 7.28\left(1 \mathrm{H}, \mathrm{t},{ }^{3} J_{\mathrm{HH}} 7.5 \mathrm{~Hz}\right.$, ArH), 3.44-3.51 (1H, dd, $\left.{ }^{3} J_{\mathrm{HH}} 7.6 \mathrm{~Hz},{ }^{2} J_{\mathrm{HH}} 17.2 \mathrm{~Hz}, \mathrm{CH}_{2} \mathrm{CH}\right), 2.60-2.82\left(2 \mathrm{H}, \mathrm{m}, \mathrm{CH}_{2} \mathrm{CH}\right), 2.49$

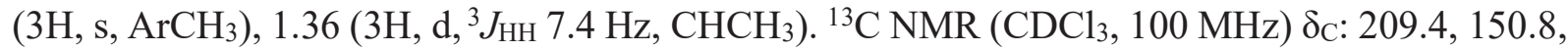
140.3, 139.1, 138.2, 136.7, 134.7, 129.1, 128.4, 128.3, 127.9, 125.5, 122.8, 42.1, 34.8, 21.4, 16.1 . EI-HRMS $(\mathrm{m} / \mathrm{z})$ calcd for $\mathrm{C}_{17} \mathrm{H}_{16} \mathrm{O}\left(\mathrm{M}^{+}\right) 236.1201$, found 236.1192

4-(2-Methoxyphenyl)-2-methyl-2,3-dihydro-1 $H$-inden-1-one $\left(\mathbf{9 a} \boldsymbol{a}_{3}\right)$. Yield, 89\%, $224 \mathrm{mg}$, colorless oil. ${ }^{1} \mathrm{H}$ NMR $\left(\mathrm{CDCl}_{3}, 400 \mathrm{MHz}\right) \delta_{\mathrm{H}}: 7.77\left(1 \mathrm{H}, \mathrm{d},{ }^{3} J_{\mathrm{HH}} 8.5 \mathrm{~Hz}, \mathrm{ArH}\right), 7.53\left(1 \mathrm{H}, \mathrm{dd},{ }^{3} J_{\mathrm{HH}}\right.$ $\left.1.2 \mathrm{~Hz},{ }^{4} J_{\mathrm{HH}} 7.4 \mathrm{~Hz}, \mathrm{ArH}\right), 7.44\left(1 \mathrm{H}, \mathrm{d},{ }^{3} J_{\mathrm{HH}} 7.5 \mathrm{~Hz}, \mathrm{ArH}\right), 7.37-7.42(1 \mathrm{H}, \mathrm{m}, \mathrm{ArH}), 7.22(1 \mathrm{H}, \mathrm{dd}$, $\left.{ }^{4} J \mathrm{HH} 1.7 \mathrm{~Hz},{ }^{3} J_{\mathrm{HH}} 7.4 \mathrm{~Hz}, \mathrm{ArH}\right), 7.06\left(1 \mathrm{H}, \mathrm{d},{ }^{3} J_{\mathrm{HH}} 8.4 \mathrm{~Hz}, \mathrm{ArH}\right), 7.02\left(1 \mathrm{H}, \mathrm{d},{ }^{3} J_{\mathrm{HH}} 8.3 \mathrm{~Hz}, \mathrm{ArH}\right)$, $3.80\left(3 \mathrm{H}, \mathrm{s}, \mathrm{OCH}_{3}\right), 3.19-3.26\left(1 \mathrm{H}, \mathrm{dd},{ }^{3} \mathrm{~J}_{\mathrm{HH}} 8.0 \mathrm{~Hz},{ }^{2} \mathrm{JHH} 17.2 \mathrm{~Hz}, \mathrm{CH}_{2} \mathrm{CH}\right), 2.67-2.71(1 \mathrm{H}, \mathrm{m}$, $\left.\mathrm{CH}_{2} \mathrm{CH}\right), 2.58\left(1 \mathrm{H}, \mathrm{dd},{ }^{3} \mathrm{~J}_{\mathrm{HH}} 4.0 \mathrm{~Hz},{ }^{2} \mathrm{~J}_{\mathrm{HH}} 17.3, \mathrm{CH}_{2} \mathrm{CH}\right), 1.28\left(3 \mathrm{H}, \mathrm{d},{ }^{3} \mathrm{~J}_{\mathrm{HH}} 7.4 \mathrm{~Hz}, \mathrm{CHCH}_{3}\right) .{ }^{13} \mathrm{C}$ $\mathrm{NMR}\left(\mathrm{CDCl}_{3}, 100 \mathrm{MHz}\right) \delta_{\mathrm{c}}: 209.8,156.4,152.8,137.5,136.2,135.8,130.8,129.4,128.0,127.4$, 122.8, 120.6, 110.9, 55.4, 42.0, 34.4, 16.2. EI-HRMS $(\mathrm{m} / \mathrm{z})$ calcd for $\mathrm{C}_{17} \mathrm{H}_{16} \mathrm{O}_{2}\left(\mathrm{M}^{+}\right) 252.1150$, found 252.1145 .

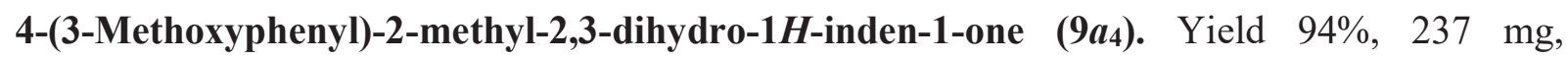
colorless oil. ${ }^{1} \mathrm{H}$ NMR $\left(\mathrm{CDCl}_{3}, 400 \mathrm{MHz}\right) \delta \mathrm{H}: 7.77\left(1 \mathrm{H}, \mathrm{d},{ }^{3} J_{\mathrm{HH}} 7.5 \mathrm{~Hz}, \mathrm{ArH}\right), 7.60\left(1 \mathrm{H}, \mathrm{d},{ }^{3} J_{\mathrm{HH}}\right.$ $7.4 \mathrm{~Hz}, \mathrm{ArH}), 7.46\left(1 \mathrm{H}, \mathrm{t},{ }^{3} J_{\mathrm{HH}} 7.5 \mathrm{~Hz}, \mathrm{ArH}\right), 7.39\left(1 \mathrm{H}, \mathrm{t},{ }^{3} J_{\mathrm{HH}} 7.9 \mathrm{~Hz}, \mathrm{ArH}\right), 7.03\left(1 \mathrm{H}, \mathrm{d},{ }^{3} J_{\mathrm{HH}} 7.6\right.$ $\mathrm{Hz}, \mathrm{ArH}), 6.98(1 \mathrm{H}, \mathrm{s}, \mathrm{ArH}), 6.95\left(1 \mathrm{H}, \mathrm{dd},{ }^{4} \mathrm{~J}_{\mathrm{HH}} 2.4 \mathrm{~Hz},{ }^{3} J_{\mathrm{HH}} 8.2 \mathrm{~Hz}, \mathrm{ArH}\right), 3.86\left(3 \mathrm{H}, \mathrm{s}, \mathrm{OCH}_{3}\right)$, 3.40-3.46 (1H, dd, $\left.{ }^{3} J_{\mathrm{HH}} 7.6 \mathrm{~Hz},{ }^{2} J_{\mathrm{HH}} 17.2 \mathrm{~Hz}, \mathrm{CH}_{2} \mathrm{CH}\right), 2.60-2.80\left(2 \mathrm{H}, \mathrm{m}, \mathrm{CH}_{2} \mathrm{CH}\right), 1.31(3 \mathrm{H}, \mathrm{d}$, $\left.{ }^{3} J_{\mathrm{HH}} 7.3 \mathrm{~Hz}, \mathrm{CHCH}_{3}\right) .{ }^{13} \mathrm{C} \mathrm{NMR}\left(\mathrm{CDCl}_{3}, 100 \mathrm{MHz}\right) \delta_{\mathrm{C}}: 209.5,159.6,150.8,140.5,140.0,136.8$, 134.7, 129.6, 127.9, 123.0, 120.9, 114.4, 112.8, 55.3, 42.1, 34.8, 16.1. EI-HRMS $(m / z):[\mathrm{M}+\mathrm{H}]^{+}$ $\mathrm{C}_{17} \mathrm{H}_{16} \mathrm{O}_{2}$, calculated 252.1150 , found 252.1146

4-(4-Methoxyphenyl)-2-methyl-2,3-dihydro-1 H-inden-1-one (9a5). Yeld, 91\%, 229 mg, white solid Mp $85-87{ }^{\circ} \mathrm{C} .{ }^{1} \mathrm{H}$ NMR $\left(\mathrm{CDCl}_{3}, 400 \mathrm{MHz}\right) \delta_{\mathrm{H}}: 7.73\left(1 \mathrm{H}, \mathrm{d},{ }^{3} J_{\mathrm{HH}} 7.6 \mathrm{~Hz}, \mathrm{ArH}\right), 7.55-7.57$ $(1 \mathrm{H}, \mathrm{m}, \mathrm{ArH}), 7.43\left(1 \mathrm{H}, \mathrm{t},{ }^{3} \mathrm{~J}_{\mathrm{HH}} 7.5 \mathrm{~Hz}, \mathrm{ArH}\right), 7.37-7.41(2 \mathrm{H}, \mathrm{m}, \mathrm{ArH}), 6.98-7.02(2 \mathrm{H}, \mathrm{m}, \mathrm{ArH})$, $3.86\left(3 \mathrm{H}, \mathrm{s}, \mathrm{OCH}_{3}\right), 3.38-3.44\left(1 \mathrm{H}, \mathrm{dd},{ }^{3} J_{\mathrm{HH}} 7.6 \mathrm{~Hz},{ }^{2} J_{\mathrm{HH}} 17.2 \mathrm{~Hz}, \mathrm{CH}_{2} \mathrm{CH}\right), 2.63-2.78(2 \mathrm{H}, \mathrm{m}$,

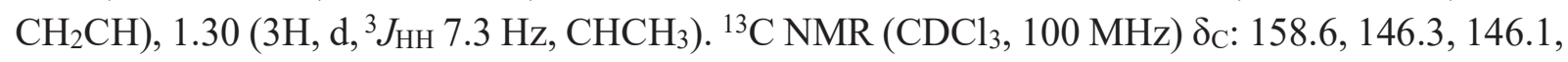
$140.6,136.9$, 133.7, 129.4, 127.1, 126.9, 124.1, 118.5, 113.7, 55.1, 42.7, 16.6. EI-HRMS $(\mathrm{m} / \mathrm{z})$ calcd for $\mathrm{C}_{17} \mathrm{H}_{16} \mathrm{O}_{2}\left(\mathrm{M}^{+}\right)$252.1150, found 252.1159

4-(3,5-Dimethylphenyl)-2-methyl-2,3-dihydro-1 $\boldsymbol{H}$-inden-1-one (9a). Yield 98\%, $245 \mathrm{mg}$, pale yellow solid, Mp $104-106{ }^{\circ} \mathrm{C} .{ }^{1} \mathrm{H} \mathrm{NMR}\left(\mathrm{CDCl}_{3}, 400 \mathrm{MHz}\right) \delta_{\mathrm{H}}: 7.75\left(1 \mathrm{H}, \mathrm{d},{ }^{3} J_{\mathrm{HH}} 8.4 \mathrm{~Hz}, \mathrm{ArH}\right)$, 7.57-7.59 (1H, m, ArH), $7.44\left(1 \mathrm{H}, \mathrm{t},{ }^{3} \mathrm{~J}_{\mathrm{HH}} 7.5 \mathrm{~Hz}, \operatorname{ArH}\right), 7.07(1 \mathrm{H}, \mathrm{s}, \mathrm{ArH}), 7.05(1 \mathrm{H}, \mathrm{s}, \mathrm{ArH})$, 3.40-3.46 (1H, dd, $\left.{ }^{3} J_{\mathrm{HH}} 7.6 \mathrm{~Hz},{ }^{2} J_{\mathrm{HH}} 17.2 \mathrm{~Hz}, \mathrm{CH}_{2} \mathrm{CH}\right), 2.65-2.80\left(2 \mathrm{H}, \mathrm{m}, \mathrm{CH}_{2} \mathrm{CH}\right), 2.40(6 \mathrm{H}, \mathrm{s}$,

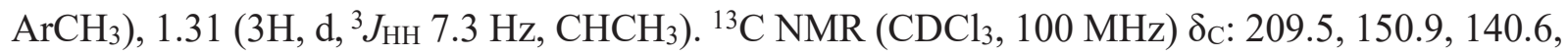
139.1, 138.1, 135.7, 134.7, 129.2, 127.8, 125.3, 122.7, 42.1, 34.9, 21.3, 16.1. EI-HRMS $(\mathrm{m} / \mathrm{z}) \mathrm{calcd}$ for $\mathrm{C}_{14} \mathrm{H}_{12} \mathrm{O}_{2}\left(\mathrm{M}^{+}\right)$250.1358, found 250.1354.

4-(4-(tert-Butyl)phenyl)-2-methyl-2,3-dihydro-1 $\boldsymbol{H}$-inden-1-one $\left(\mathbf{9} \boldsymbol{a}_{7}\right)$. Yield: $94 \%, 261 \mathrm{mg}$, white solid, Mp $102-104{ }^{\circ} \mathrm{C} .{ }^{1} \mathrm{H}$ NMR $\left(\mathrm{CDCl}_{3}, 400 \mathrm{MHz}\right) \delta_{\mathrm{H}}: 7.76\left(1 \mathrm{H}, \mathrm{d},{ }^{3} J_{\mathrm{HH}} 7.4 \mathrm{~Hz}, \mathrm{ArH}\right)$, 
$7.60\left(1 \mathrm{H}, \mathrm{dd},{ }^{3} J_{\mathrm{HH}} 7.6 \mathrm{~Hz},{ }^{4} J_{\mathrm{HH}} 1.2 \mathrm{~Hz}, \mathrm{ArH}\right), 7.36-7.54(5 \mathrm{H}, \mathrm{m}, \mathrm{ArH}), 3.43-3.49\left(1 \mathrm{H}, \mathrm{dd},{ }^{3} J_{\mathrm{HH}} 7.6\right.$ $\left.\mathrm{Hz},{ }^{2} J_{\mathrm{HH}} 17.2 \mathrm{~Hz}, \mathrm{CH}_{2} \mathrm{CH}\right), 2.80\left(1 \mathrm{H}, \mathrm{dd},{ }^{3} J_{\mathrm{HH}} 4.0 \mathrm{~Hz},{ }^{2} J_{\mathrm{HH}} 17.2 \mathrm{~Hz}, \mathrm{CH}_{2} \mathrm{CH}\right), 2.66-2.76(\mathrm{H}, \mathrm{m}$,

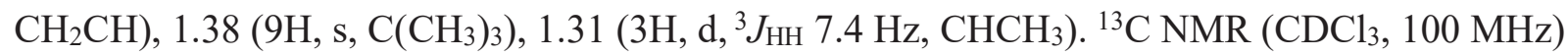
$\delta_{\mathrm{C}}: 209.5,150.9,150.8,140.1,136.8,136.2,134.7,128.1,127.9,125.5,122.7,42.2,34.9,34.6$, 31.3, 16.1. EI-HRMS $(\mathrm{m} / \mathrm{z})$ calcd for $\mathrm{C}_{20} \mathrm{H}_{22} \mathrm{O}\left(\mathrm{M}^{+}\right) 278.1671$, found 278.1661

2-Methyl-4-(4-(trifluoromethoxy)phenyl)-2,3-dihydro-1H-inden-1-one (9a8). Yield, 90\%, 275 mg, colorless oil. ${ }^{1} \mathrm{H} \mathrm{NMR}\left(\mathrm{CDCl}_{3}, 400 \mathrm{MHz}\right) \delta_{\mathrm{H}}: 7.79\left(1 \mathrm{H}, \mathrm{d},{ }^{3} J_{\mathrm{HH}} 8.5 \mathrm{~Hz}, \mathrm{ArH}\right), 7.58(1 \mathrm{H}, \mathrm{dd}$, $\left.{ }^{4} J_{\mathrm{HH}} 1.2 \mathrm{~Hz},{ }^{3} J_{\mathrm{HH}} 7.5 \mathrm{~Hz}, \mathrm{ArH}\right), 7.46-7.50(3 \mathrm{H}, \mathrm{m}, \mathrm{ArH}), 7.33\left(2 \mathrm{H}, \mathrm{d},{ }^{3} J_{\mathrm{HH}} 8.7 \mathrm{~Hz}, \mathrm{ArH}\right), 3.37-$ $3.43\left(1 \mathrm{H}, \mathrm{dd},{ }^{3} J_{\mathrm{HH}} 8.0 \mathrm{~Hz},{ }^{2} J_{\mathrm{HH}} 17.6 \mathrm{~Hz}, \mathrm{CH}_{2} \mathrm{CH}\right), 2.70-2.78\left(2 \mathrm{H}, \mathrm{m}, \mathrm{CH}_{2} \mathrm{CH}\right), 1.32\left(3 \mathrm{H}, \mathrm{d},{ }^{3} J_{\mathrm{HH}}\right.$

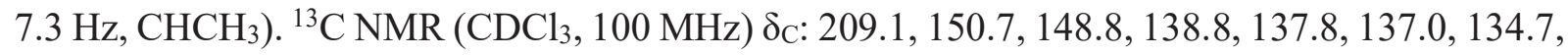
$129.9,128.1,123.4,121.1,120.5\left(\mathrm{q},{ }^{1} \mathrm{JFC}=255.9 \mathrm{~Hz}\right) 42.2,34.8,16.1$. EI-HRMS $(\mathrm{m} / \mathrm{z}) \mathrm{calcd}$ for $\mathrm{C}_{17} \mathrm{H}_{13} \mathrm{~F}_{3} \mathrm{O}_{2}\left(\mathrm{M}^{+}\right) 306.0868$, found 306.0872

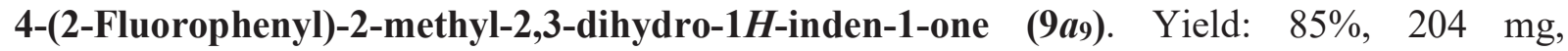
colorless oil. ${ }^{1} \mathrm{H}$ NMR $\left(\mathrm{CDCl}_{3}, 400 \mathrm{MHz}\right) \delta_{\mathrm{H}}: 7.80\left(1 \mathrm{H}, \mathrm{d},{ }^{3} J_{\mathrm{HH}} 7.6 \mathrm{~Hz}, \mathrm{ArH}\right), 7.56\left(1 \mathrm{H}, \mathrm{d},{ }^{3} J_{\mathrm{HH}}\right.$ $7.5 \mathrm{~Hz}, \mathrm{ArH}), 7.44-7.48$ (1H, m, ArH), 7.37-7.43 (1H, m, ArH), 7.31-7.35 (1H, m, ArH), 7.10$7.30(2 \mathrm{H}, \mathrm{m}, \mathrm{ArH}), 3.25-3.31\left(1 \mathrm{H}, \mathrm{dd},{ }^{3} J_{\mathrm{HH}} 7.6 \mathrm{~Hz},{ }^{2} J_{\mathrm{HH}} 17.2 \mathrm{~Hz}, \mathrm{CH}_{2} \mathrm{CH}\right), 2.60-2.80(2 \mathrm{H}, \mathrm{m}$, $\left.\mathrm{CH}_{2} \mathrm{CH}\right), 1.29\left(3 \mathrm{H}, \mathrm{d},{ }^{3} \mathrm{~J}_{\mathrm{HH}} 7.3 \mathrm{~Hz}, \mathrm{CHCH}_{3}\right) .{ }^{13} \mathrm{C} \mathrm{NMR}\left(\mathrm{CDCl}_{3}, 100 \mathrm{MHz}\right) \delta_{\mathrm{C}}: 209.3,169.5(\mathrm{~d}$, $\left.{ }^{1} J \mathrm{FC} 245.5 \mathrm{~Hz}\right), 152.3,136.7,135.7,134.6,131.2\left(\mathrm{~d},{ }^{4} J \mathrm{FC} 3.6 \mathrm{~Hz}\right), 129.9\left(\mathrm{~d},{ }^{3} J \mathrm{FC} 8.0 \mathrm{~Hz}\right), 127.7$, $126.6\left(\mathrm{~d},{ }^{2} J \mathrm{FC} 16.0 \mathrm{~Hz}\right), 124.3\left(\mathrm{~d},{ }^{4} J \mathrm{FC} 3.7 \mathrm{~Hz}\right), 123.6,115.9\left(\mathrm{~d},{ }^{2} J \mathrm{FC} 22.2 \mathrm{~Hz}\right), 42.0,34.2,16.1$. EI-HRMS $(\mathrm{m} / \mathrm{z})$ calcd for $\mathrm{C}_{16} \mathrm{H}_{13} \mathrm{FO}\left(\mathrm{M}^{+}\right) 240.0950$, found 240.0943

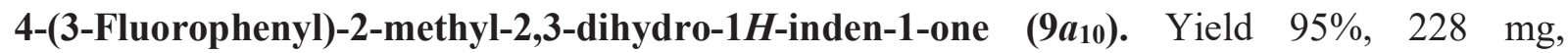
colorless oil. ${ }^{1} \mathrm{H}$ NMR $\left(\mathrm{CDCl}_{3}, 400 \mathrm{MHz}\right) \delta_{\mathrm{H}}: 7.78\left(1 \mathrm{H}, \mathrm{d},{ }^{3} J_{\mathrm{HH}} 7.6 \mathrm{~Hz}, \mathrm{ArH}\right), 7.58\left(1 \mathrm{H}, \mathrm{d},{ }^{3} J_{\mathrm{HH}}\right.$ $7.4 \mathrm{~Hz}, \mathrm{ArH}), 7.40-7.48(2 \mathrm{H}, \mathrm{m}, \mathrm{ArH}), 7.23\left(1 \mathrm{H}, \mathrm{d},{ }^{3} J_{\mathrm{HH}} 7.7 \mathrm{~Hz}, \mathrm{ArH}\right), 7.13-7.17(1 \mathrm{H}, \mathrm{m}, \mathrm{ArH})$, 7.06-7.11 $(1 \mathrm{H}, \mathrm{m}, \mathrm{ArH}), 3.37-3.43\left(1 \mathrm{H}, \mathrm{dd},{ }^{3} J_{\mathrm{HH}} 7.6 \mathrm{~Hz},{ }^{2} J_{\mathrm{HH}} 16.8 \mathrm{~Hz}, \mathrm{CH}_{2} \mathrm{CH}\right), 2.65-2.80(2 \mathrm{H}$, $\left.\mathrm{m}, \mathrm{CH}_{2} \mathrm{CH}\right), 1.31\left(3 \mathrm{H}, \mathrm{d},{ }^{3} \mathrm{~J}_{\mathrm{HH}} 7.3 \mathrm{~Hz}, \mathrm{CHCH}_{3}\right) .{ }^{13} \mathrm{C} \mathrm{NMR}\left(\mathrm{CDCl}_{3}, 100 \mathrm{MHz}\right) \delta_{\mathrm{C}}: 209.1,162.8(\mathrm{~d}$, $\left.{ }^{1} J \mathrm{FC} 245.1 \mathrm{~Hz}\right), 150.7,141.3$ (d, $\left.{ }^{3} J \mathrm{FC} 7.6 \mathrm{~Hz}\right), 139.0,137.0,134.7,130.2\left(\mathrm{~d},{ }^{3} J \mathrm{FC} 8.4 \mathrm{~Hz}\right), 128.1$, $124.3\left(\mathrm{~d},{ }^{4} J \mathrm{FC} 2.9 \mathrm{~Hz}\right), 123.5,115.5$ (d, $\left.{ }^{2} J \mathrm{FC} 21.6 \mathrm{~Hz}\right), 114.6(\mathrm{~d}, 2 J \mathrm{FC} 20.9 \mathrm{~Hz}), 42.1,34.8,16.1$. EI-HRMS $(\mathrm{m} / \mathrm{z})$ calcd for $\mathrm{C}_{16} \mathrm{H}_{13} \mathrm{FO}\left(\mathrm{M}^{+}\right) 240.0950$, found 240.0946

4-(4-Fluorophenyl)-2-methyl-2,3-dihydro-1H-inden-1-one (9a11). Yield 97\%, $232 \mathrm{mg}$, colorless oil. ${ }^{1} \mathrm{H}$ NMR $\left(\mathrm{CDCl}_{3}, 400 \mathrm{MHz}\right) \delta_{\mathrm{H}}: 7.76\left(1 \mathrm{H}, \mathrm{d},{ }^{3} J_{\mathrm{HH}} 7.5 \mathrm{~Hz}, \mathrm{ArH}\right), 7.56\left(1 \mathrm{H}, \mathrm{d},{ }^{3} J_{\mathrm{HH}}\right.$ $7.4 \mathrm{~Hz}, \mathrm{ArH}), 7.40-7.46(3 \mathrm{H}, \mathrm{m}, \mathrm{ArH}), 7.15\left(2 \mathrm{H}, \mathrm{t},{ }^{3} J_{\mathrm{HH}} 8.7 \mathrm{~Hz}, \mathrm{ArH}\right), 3.35-3.41\left(1 \mathrm{H}, \mathrm{dd},{ }^{3} J_{\mathrm{HH}} 7.6\right.$ $\left.\mathrm{Hz},{ }^{2} J_{\mathrm{HH}} 16.8 \mathrm{~Hz}, \mathrm{CH}_{2} \mathrm{CH}\right), 2.68-2.76\left(2 \mathrm{H}, \mathrm{m}, \mathrm{CH}_{2} \mathrm{CH}\right), 1.31\left(3 \mathrm{H}, \mathrm{d},{ }^{3} J_{\mathrm{HH}} 7.3 \mathrm{~Hz}, \mathrm{CHCH}_{3}\right) .{ }^{13} \mathrm{C}$ NMR $\left(\mathrm{CDCl}_{3}, 100 \mathrm{MHz}\right) \delta_{\mathrm{C}}: 209.2,162.4\left(\mathrm{~d},{ }^{1} J \mathrm{FC} 245.6 \mathrm{~Hz}\right), 150.8,139.2,136.9,135.2\left(\mathrm{~d},{ }^{4} J \mathrm{FC}\right.$ $3.4 \mathrm{~Hz}$ ), 134.7, 130.1 (d, $\left.{ }^{3} J \mathrm{FC} 8.1 \mathrm{~Hz}\right), 130.0,128.0,123.1,115.6$ (d, $\left.{ }^{2} J \mathrm{FC} 21.3 \mathrm{~Hz}\right), 42.1,34.8$, 16.1. EI-HRMS $(\mathrm{m} / \mathrm{z})$ calcd for $\mathrm{C}_{16} \mathrm{H}_{13} \mathrm{FO}\left(\mathrm{M}^{+}\right) 240.0950$, found 240.0953

4-(4-Chlorophenyl)-2-methyl-2,3-dihydro-1H-inden-1-one (9a12). Yield 90\%, $230 \mathrm{mg}$, white solid, Mp $83-85^{\circ} \mathrm{C} .{ }^{1} \mathrm{H}$ NMR $\left(\mathrm{CDCl}_{3}, 400 \mathrm{MHz}\right) \delta_{\mathrm{H}}: 7.78\left(1 \mathrm{H}, \mathrm{d},{ }^{3} J_{\mathrm{HH}} 8.4 \mathrm{~Hz}, \mathrm{ArH}\right), 7.57(1 \mathrm{H}$, dd, $\left.{ }^{4} J_{\mathrm{HH}} 1.1 \mathrm{~Hz},{ }^{3} J_{\mathrm{HH}} 7.4 \mathrm{~Hz}, \mathrm{ArH}\right), 7.43-7.49$ (3H, m, ArH), 7.37-7.40 (2H, m, ArH), 3.35-3.42 $\left(1 \mathrm{H}, \mathrm{dd},{ }^{3} J_{\mathrm{HH}} 8.4 \mathrm{~Hz},{ }^{2} J_{\mathrm{HH}} 17.6 \mathrm{~Hz}, \mathrm{CH}_{2} \mathrm{CH}\right), 2.69-2.77\left(2 \mathrm{H}, \mathrm{m}, \mathrm{CH}_{2} \mathrm{CH}\right), 1.31\left(3 \mathrm{H}, \mathrm{d},{ }^{3} J_{\mathrm{HH}} 7.3 \mathrm{~Hz}\right.$, $\left.\mathrm{CHCH}_{3}\right) .{ }^{13} \mathrm{CNMR}\left(\mathrm{CDCl}_{3}, 100 \mathrm{MHz}\right) \delta_{\mathrm{C}}: 209.2,150.7,139.0,137.6,137.0,134.6,133.8,129.8$, 
128.8, 128.1, 123.3, 42.2, 34.8, 16.1. EI-HRMS $(\mathrm{m} / \mathrm{z})$ calcd for $\mathrm{C}_{16} \mathrm{H}_{13} \mathrm{ClO}\left(\mathrm{M}^{+}\right)$256.0655, found 256.0652 .

2-Methyl-4-(3-(trifluoromethyl)phenyl)-2,3-dihydro-1 $\boldsymbol{H}$-inden-1-one (9a13). Yield 87\%, 252 mg, white solid, Mp $114-116{ }^{\circ} \mathrm{C} .{ }^{1} \mathrm{H}$ NMR $\left(\mathrm{CDCl}_{3}, 400 \mathrm{MHz}\right) \delta_{\mathrm{H}}: 7.82\left(1 \mathrm{H}, \mathrm{d},{ }^{3} J_{\mathrm{HH}} 7.6 \mathrm{~Hz}, \mathrm{ArH}\right)$, $7.72(1 \mathrm{H}, \mathrm{s}, \mathrm{ArH}), 7.66\left(2 \mathrm{H}, \mathrm{d},{ }^{3} J_{\mathrm{HH}} 7.4 \mathrm{~Hz}, \mathrm{ArH}\right), 7.59-7.62(2 \mathrm{H}, \mathrm{m}, \mathrm{ArH}), 7.50\left(1 \mathrm{H}, \mathrm{t},{ }^{3} J_{\mathrm{HH}} 7.5\right.$ $\mathrm{Hz}, \mathrm{ArH}), 3.37-3.44\left(1 \mathrm{H}, \mathrm{dd},{ }^{3} \mathrm{~J}_{\mathrm{HH}} 8.8 \mathrm{~Hz},{ }^{2} \mathrm{~J}_{\mathrm{HH}} 18.4 \mathrm{~Hz}, \mathrm{CH}_{2} \mathrm{CH}\right), 2.70-2.81\left(2 \mathrm{H}, \mathrm{m}, \mathrm{CH}_{2} \mathrm{CH}\right)$,

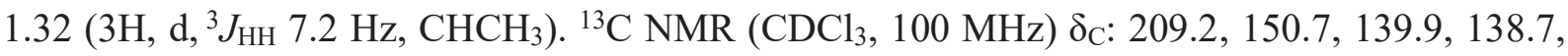
137.0, 134.8, 131.8, 129.1, 128.2, $125.3\left(\mathrm{q},{ }^{4} J \mathrm{FC}=3.7 \mathrm{~Hz}\right), 124.5\left(\mathrm{q},{ }^{4} J \mathrm{FC}=3.9 \mathrm{~Hz}\right), 123.7,42.2$, 34.6, 16.1. EI-HRMS $(\mathrm{m} / \mathrm{z})$ calcd for $\mathrm{C}_{17} \mathrm{H}_{13} \mathrm{~F}_{3} \mathrm{O}\left(\mathrm{M}^{+}\right) 290.0918$, found 290.0928

2-Methyl-4-(4-(trifluoromethyl)phenyl)-2,3-dihydro-1 $\boldsymbol{H}$-inden-1-one (6a $\left.\boldsymbol{a}_{14}\right)$. Yield 90\%, 261 mg, white solid, Mp $93-95{ }^{\circ} \mathrm{C} .{ }^{1} \mathrm{H}$ NMR $\left(\mathrm{CDCl}_{3}, 400 \mathrm{MHz}\right) \delta_{\mathrm{H}}: 7.82\left(1 \mathrm{H}, \mathrm{d},{ }^{3} J_{\mathrm{HH}} 7.5 \mathrm{~Hz}, \mathrm{ArH}\right)$, $7.74\left(2 \mathrm{H}, \mathrm{d},{ }^{3} J_{\mathrm{HH}} 8.0 \mathrm{~Hz}, \mathrm{ArH}\right), 7.57-7.61(3 \mathrm{H}, \mathrm{m}, \mathrm{ArH}), 7.50\left(1 \mathrm{H}, \mathrm{t},{ }^{3} J_{\mathrm{HH}} 7.5 \mathrm{~Hz}, \mathrm{ArH}\right), 3.37-3.43$ $\left(1 \mathrm{H}, \mathrm{dd},{ }^{3} J_{\mathrm{HH}} 7.6 \mathrm{~Hz},{ }^{2} J_{\mathrm{HH}} 18.0 \mathrm{~Hz}, \mathrm{CH}_{2} \mathrm{CH}\right), 2.71-2.79\left(2 \mathrm{H}, \mathrm{m}, \mathrm{CH}_{2} \mathrm{CH}\right), 1.32\left(3 \mathrm{H}, \mathrm{d},{ }^{3} J_{\mathrm{HH}} 7.2 \mathrm{~Hz}\right.$, $\left.\mathrm{CHCH}_{3}\right) .{ }^{13} \mathrm{C} \mathrm{NMR}\left(\mathrm{CDCl}_{3}, 100 \mathrm{MHz}\right) \delta_{\mathrm{c}}: 209.0,150.7,142.8,138.8,137.1,134.7,129.9\left(\mathrm{q},{ }^{2} J \mathrm{FC}\right.$ $32.5 \mathrm{~Hz}$ ), 128.9, 128.2, 125.6 (q, ${ }^{4} J \mathrm{FC} 3.6 \mathrm{~Hz}$ ), 124.1 (q, $\left.{ }^{1} J \mathrm{FC} 270.4 \mathrm{~Hz}\right), 123.8,42.2,34.7,16.1$. EI-HRMS $(\mathrm{m} / \mathrm{z})$ calcd for $\mathrm{C}_{17} \mathrm{H}_{3} \mathrm{~F}_{3} \mathrm{O}\left(\mathrm{M}^{+}\right) 290.0918$, found 290.0924

4-(3,5-bis(trifluoromethyl)phenyl)-2-methyl-2,3-dihydro-1H-inden-1-one $\left(\mathbf{9} \boldsymbol{a}_{15}\right)$. Yield 98\%, $255 \mathrm{mg}$, white solid, Mp $115-117{ }^{\circ} \mathrm{C} .{ }^{1} \mathrm{H}$ NMR $\left(\mathrm{CDCl}_{3}, 400 \mathrm{MHz}\right) \delta_{\mathrm{H}}: 7.92(3 \mathrm{H}, \mathrm{s}, \mathrm{ArH}), 7.86$ $\left(1 \mathrm{H}, \mathrm{d},{ }^{3} J_{\mathrm{HH}} 7.6 \mathrm{~Hz}, \mathrm{ArH}\right), 7.63\left(1 \mathrm{H}, \mathrm{d},{ }^{3} J_{\mathrm{HH}} 7.2 \mathrm{~Hz}, \mathrm{ArH}\right), 7.54\left(1 \mathrm{H}, \mathrm{t},{ }^{3} J_{\mathrm{HH}} 7.6 \mathrm{~Hz}, \mathrm{ArH}\right), 3.37-$ $3.43\left(1 \mathrm{H}, \mathrm{dd},{ }^{3} J_{\mathrm{HH}} 8.0 \mathrm{~Hz},{ }^{2} \mathrm{~J}_{\mathrm{HH}} 17.2 \mathrm{~Hz}, \mathrm{CH}_{2} \mathrm{CH}\right), 2.71-2.79\left(2 \mathrm{H}, \mathrm{m}, \mathrm{CH}_{2} \mathrm{CH}\right), 1.33\left(3 \mathrm{H}, \mathrm{d},{ }^{3} \mathrm{~J}_{\mathrm{HH}}\right.$ $\left.7.6 \mathrm{~Hz}, \mathrm{CHCH}_{3}\right) .{ }^{13} \mathrm{C} \mathrm{NMR}\left(\mathrm{CDCl}_{3}, 100 \mathrm{MHz}\right) \delta_{\mathrm{C}}: 208.5,150.5,141.3,137.4,137.2,134.7,132.2$ (q ${ }^{2} J$ FC $\left.33.2 \mathrm{~Hz}\right), 128.6(\mathrm{~m}), 128.5,123.3$ (q $\left.{ }^{1} J \mathrm{FC} 271.0 \mathrm{~Hz}\right), 124.5,121.5(\mathrm{~m}), 42.2,34.5,16.1$. EI-HRMS $(\mathrm{m} / z)$ calcd for $\mathrm{C}_{18} \mathrm{H}_{12} \mathrm{~F}_{6} \mathrm{O}\left(\mathrm{M}^{+}\right) 358.0792$, found 358.0796

-(2-Methyl-1-oxo-2,3-dihydro-1H-inden-4-yl)benzonitrile $\left(\mathbf{9 a}_{16}\right)$. Yield 90\%, $222 \mathrm{mg}$, white solid, Mp 160 - $162{ }^{\circ} \mathrm{C} .{ }^{1} \mathrm{H}$ NMR $\left(\mathrm{CDCl}_{3}, 400 \mathrm{MHz}\right) \delta_{\mathrm{H}:} 7.81\left(1 \mathrm{H}, \mathrm{d},{ }^{3} J_{\mathrm{HH}} 7.2 \mathrm{~Hz}, \mathrm{ArH}\right), 7.64-7.75$ $(5 \mathrm{H}, \mathrm{m}, \mathrm{ArH}), 7.50\left(1 \mathrm{H}, \mathrm{t},{ }^{3} J_{\mathrm{HH}} 7.2 \mathrm{~Hz}, \mathrm{ArH}\right), 3.34-3.41\left(1 \mathrm{H}, \mathrm{dd},{ }^{3} J_{\mathrm{HH}} 8.8 \mathrm{~Hz},{ }^{2} J_{\mathrm{HH}} 18.0 \mathrm{~Hz}\right.$, $\left.\mathrm{CH}_{2} \mathrm{CH}\right), 2.72-2.77\left(2 \mathrm{H}, \mathrm{m}, \mathrm{CH}_{2} \mathrm{CH}\right), 1.31\left(3 \mathrm{H}, \mathrm{d},{ }^{3} \mathrm{~J}_{\mathrm{HH}} 7.2 \mathrm{~Hz}, \mathrm{CHCH}_{3}\right) .{ }^{13} \mathrm{C} \mathrm{NMR}\left(\mathrm{CDCl}_{3}, 100\right.$ MHz) $\delta_{\mathrm{C}}: 208.8,150.5,140.5,137.8,137.2,134.6,132.9,131.9,131.2,129.6,128.3,124.0,118.5$, 113.0, 42.2, 34.6, 16.1. EI-HRMS $(\mathrm{m} / \mathrm{z})$ calcd for $\mathrm{C}_{17} \mathrm{H}_{13} \mathrm{NO}\left(\mathrm{M}^{+}\right)$247.0997, found 247.0996.

4-(2-Methyl-1-oxo-2,3-dihydro-1 $\boldsymbol{H}$-inden-4-yl)benzonitrile $\left(\boldsymbol{9 a}_{\mathbf{1 7}}\right)$. Yield $96 \%, 237 \mathrm{mg}$, white solid, mp $112-114{ }^{\circ} \mathrm{C} .{ }^{1} \mathrm{H}$ NMR $\left(\mathrm{CDCl}_{3}, 400 \mathrm{MHz}\right) \delta_{\mathrm{H}}: 7.81\left(1 \mathrm{H}, \mathrm{d},{ }^{3} J_{\mathrm{HH}} 7.2 \mathrm{~Hz}, \mathrm{ArH}\right), 7.77(2 \mathrm{H}$, d, $\left.{ }^{3} J_{\mathrm{HH}} 8.4 \mathrm{~Hz}, \mathrm{ArH}\right), 7.57-7.60(3 \mathrm{H}, \mathrm{m}, \mathrm{ArH}), 7.50\left(1 \mathrm{H}, \mathrm{t},{ }^{3} J_{\mathrm{HH}} 7.2 \mathrm{~Hz}, \operatorname{ArH}\right), 3.35-3.42(1 \mathrm{H}, \mathrm{dd}$, $\left.{ }^{3} J_{\mathrm{HH}} 8.8 \mathrm{~Hz},{ }^{2} J_{\mathrm{HH}} 18.0 \mathrm{~Hz}, \mathrm{CH}_{2} \mathrm{CH}\right), 2.68-2.77\left(2 \mathrm{H}, \mathrm{m}, \mathrm{CH}_{2} \mathrm{CH}\right), 1.31\left(3 \mathrm{H}, \mathrm{d},{ }^{3} J_{\mathrm{HH}} 7.2 \mathrm{~Hz}, \mathrm{CHCH}_{3}\right)$. ${ }^{13} \mathrm{C} \mathrm{NMR}\left(\mathrm{CDCl}_{3}, 100 \mathrm{MHz}\right) \delta_{\mathrm{C}}: 208.7,150.4,143.8,138.2,137.1,134.5,132.4,129.2,128.2$, 124.0, 118.5, 111.5, 42.1, 34.6, 16.9. EI-HRMS $(m / z)$ calcd for $\mathrm{C}_{17} \mathrm{H}_{13} \mathrm{NO}\left(\mathrm{M}^{+}\right)$247.0997, found 247.0995 .

4-([1,1'-Biphenyl]-4-yl)-2-methyl-2,3-dihydro-1H-inden-1-one (9a18). Yield 82\%, $244 \mathrm{mg}$, colorless oil. ${ }^{1} \mathrm{H} \mathrm{NMR}\left(\mathrm{CDCl}_{3}, 400 \mathrm{MHz}\right) \delta_{\mathrm{H}}: 7.81\left(1 \mathrm{H}, \mathrm{d},{ }^{3} J_{\mathrm{HH}} 7.6 \mathrm{~Hz}, \mathrm{ArH}\right), 7.71-7.74(2 \mathrm{H}, \mathrm{m}$, ArH), 7.65-7.69 (3H, m, ArH), 7.55-7.57 (2H, m, ArH), 7.47-7.51 (3H, m, ArH), 7.37-7.42 (1H, m, ArH), 3.46-3.52 (1H, dd, $\left.{ }^{3} J_{\mathrm{HH}} 8.0 \mathrm{~Hz},{ }^{2} J_{\mathrm{HH}} 17.2 \mathrm{~Hz}, \mathrm{CH}_{2} \mathrm{CH}\right), 2.84\left(1 \mathrm{H}, \mathrm{dd},{ }^{3} J_{\mathrm{HH}} 4.1 \mathrm{~Hz},{ }^{2} J_{\mathrm{HH}}\right.$ 
$\left.17.2 \mathrm{~Hz}, \mathrm{CH}_{2} \mathrm{CH}\right), 2.73-2.76\left(2 \mathrm{H}, \mathrm{m}, \mathrm{CH}_{2} \mathrm{CH}\right), 1.34\left(3 \mathrm{H}, \mathrm{d},{ }^{3} \mathrm{~J}_{\mathrm{HH}} 7.6 \mathrm{~Hz}, \mathrm{CHCH}_{3}\right) .{ }^{13} \mathrm{C} \mathrm{NMR}$ $\left(\mathrm{CDCl}_{3}, 100 \mathrm{MHz}\right) \delta_{\mathrm{C}}: 209.3,150.8,140.4,140.4,139.7,138.0,136.9,134.7,128.9,128.8,128.0$, 127.5, 127.2, 127.0, 123.0, 42.1, 34.9, 16.1. EI-HRMS $(\mathrm{m} / z)$ calcd for $\mathrm{C}_{22} \mathrm{H}_{18} \mathrm{O}\left(\mathrm{M}^{+}\right)$298.1358, found 298.1369

4-(2-Chlorophenyl)-2-methyl-2,3-dihydro-1H-inden-1-one $\quad\left(\boldsymbol{9 a}_{\mathbf{1 9}}\right)$. Yield $\mathbf{9 0 \%}, 230 \mathrm{mg}$, colorless oil. ${ }^{1} \mathrm{H}$ NMR $\left(\mathrm{CDCl}_{3}, 400 \mathrm{MHz}\right) \delta_{\mathrm{H}}: 7.75-7.82\left(1 \mathrm{H}, \mathrm{dd},{ }^{3} J_{\mathrm{HH}} 7.2 \mathrm{~Hz},{ }^{4} J_{\mathrm{HH}} 1.6 \mathrm{~Hz}, \mathrm{ArH}\right)$, 7.44-7.58 (3H, m, ArH), 7.33-7.38 (2H, m, ArH), 7.26-7.30 (1H, m, ArH), 3.18-3.43 (H, br, $\left.\mathrm{CH}_{2} \mathrm{CH}\right), 2.65-2.80\left(\mathrm{H}, \mathrm{m}, \mathrm{CH}_{2} \mathrm{CH}\right), 2.45-2.650\left(\mathrm{H}, \mathrm{br}, \mathrm{CH}_{2} \mathrm{CH}\right), 1.28\left(3 \mathrm{H}, \mathrm{d},{ }^{3} \mathrm{JHH}_{\mathrm{HH}} 7.2 \mathrm{~Hz}, \mathrm{CHCH}_{3}\right)$. ${ }^{13} \mathrm{C} \mathrm{NMR}\left(\mathrm{CDCl}_{3}, 100 \mathrm{MHz}\right) \delta_{\mathrm{C}}: 209.3,152.1,137.9,135.4,133.2,130.8,129.8,129.3,127.5$, 126.8, 123.5, 42.0, 34.1, 16.2. EI-HRMS $(\mathrm{m} / z)$ calcd for $\mathrm{C}_{16} \mathrm{H}_{13} \mathrm{ClO}\left(\mathrm{M}^{+}\right) 256.0655$, found 256.0652

4-(3-Chlorophenyl)-2-methyl-2,3-dihydro-1H-inden-1-one $\quad\left(\boldsymbol{9 a}_{\mathbf{2 0}}\right)$. Yield 87\%, $222 \mathrm{mg}$, colorless oil. ${ }^{1} \mathrm{H}$ NMR $\left(\mathrm{CDCl}_{3}, 400 \mathrm{MHz}\right) \delta_{\mathrm{H}}: 7.79\left(1 \mathrm{H}, \mathrm{d},{ }^{3} J_{\mathrm{HH}} 7.2 \mathrm{~Hz}, \mathrm{ArH}\right), 7.57\left(1 \mathrm{H}, \mathrm{dd},{ }^{3} J_{\mathrm{HH}}\right.$ $\left.7.6 \mathrm{~Hz},{ }^{4} J_{\mathrm{HH}} 1.2 \mathrm{~Hz}, \mathrm{ArH}\right), 7.32-7.49(5 \mathrm{H}, \mathrm{m}, \mathrm{ArH}), 3.37-3.43\left(1 \mathrm{H}, \mathrm{dd},{ }^{3} J_{\mathrm{HH}} 8.8 \mathrm{~Hz},{ }^{2} J_{\mathrm{HH}} 18.0 \mathrm{~Hz}\right.$, $\left.\mathrm{CH}_{2} \mathrm{CH}\right), 2.68-2.78\left(2 \mathrm{H}, \mathrm{m}, \mathrm{CH}_{2} \mathrm{CH}\right), 1.31\left(3 \mathrm{H}, \mathrm{d},{ }^{3} \mathrm{~J}_{\mathrm{HH}} 7.2 \mathrm{~Hz}, \mathrm{CHCH}_{3}\right) .{ }^{13} \mathrm{C} \mathrm{NMR}\left(\mathrm{CDCl}_{3}, 100\right.$ MHz) $\delta_{\mathrm{C}}: 209.1,150.7,140.9,138.8,137.0,134.7,134.5,129.876,128.6,128.1,127.8,126.7$, 123.5, 42.2, 34.7, 16.1 . EI-HRMS $(\mathrm{m} / z)$ calcd for $\mathrm{C}_{16} \mathrm{H}_{13} \mathrm{ClO}\left(\mathrm{M}^{+}\right) 256.0655$, found 256.0652

2-Methyl-4-(3-nitrophenyl)-2,3-dihydro-1H-inden-1-one $\left(\mathbf{9} \boldsymbol{a}_{\mathbf{2 1}}\right)$. Yield 80\%, $213 \mathrm{mg}$, white solid, mp $111-113{ }^{\circ} \mathrm{C} .{ }^{1} \mathrm{H} \mathrm{NMR}\left(\mathrm{CDCl}_{3}, 400 \mathrm{MHz}\right) \delta_{\mathrm{H}}: 8.35\left(1 \mathrm{H}, \mathrm{t},{ }^{3} J_{\mathrm{HH}} 2.0 \mathrm{~Hz}, \mathrm{ArH}\right), 8.26-8.29$ $(1 \mathrm{H}, \mathrm{m}, \mathrm{ArH}), 7.85\left(1 \mathrm{H}, \mathrm{d},{ }^{3} J_{\mathrm{HH}} 7.6 \mathrm{~Hz}, \mathrm{ArH}\right), 7.80\left(1 \mathrm{H}, \mathrm{d},{ }^{3} J_{\mathrm{HH}} 7.6 \mathrm{~Hz}, \mathrm{ArH}\right), 7.61-7.74(2 \mathrm{H}, \mathrm{m}$, $\mathrm{ArH}), 7.53\left(1 \mathrm{H}, \mathrm{t},{ }^{3} J_{\mathrm{HH}} 7.5 \mathrm{~Hz}, \mathrm{ArH}\right), 3.38-3.45\left(1 \mathrm{H}, \mathrm{dd},{ }^{3} J_{\mathrm{HH}} 8.8 \mathrm{~Hz},{ }^{2} J_{\mathrm{HH}} 18.0 \mathrm{~Hz}, \mathrm{CH}_{2} \mathrm{CH}\right), 2.74-$

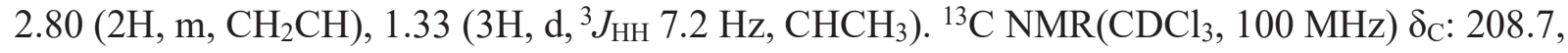
$150.6,140.8,137.7,137.3,134.7,134.5,129.7,128.4,124.2,123.4,122.6,42.2$, 34.6, 16.1. EIHRMS $(m / z)$ calcd for $\mathrm{C}_{16} \mathrm{H}_{13} \mathrm{NO}_{3}\left(\mathrm{M}^{+}\right)$267.0895, found 267.0889 .

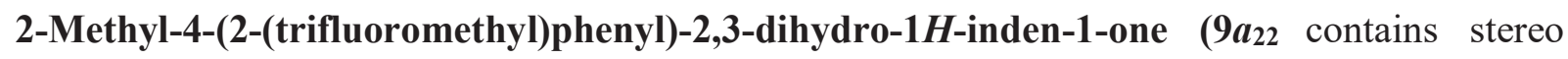
isomers (1 : 1)). Yield 79\%, $229 \mathrm{mg}$, colorless oil. ${ }^{1} \mathrm{H} \mathrm{NMR}\left(\mathrm{CDCl}_{3}, 400 \mathrm{MHz}\right) \delta_{\mathrm{H}}: 7.76-7.85(2 \mathrm{H}$, ArH), 7.58-7.65 (1H, ArH), 7.52-7.58 (1H, ArH), 7.41-7.48 (2H, m, ArH), 7.27-7.30 (1H, ArH), 3.97-3.11 ( $\left.\mathrm{H}, \mathrm{m}, \mathrm{CH}_{2} \mathrm{CH}\right), 2.66-2.70\left(\mathrm{H}, \mathrm{m}, \mathrm{CH}_{2} \mathrm{CH}\right), 2.33-2.64\left(\mathrm{H}, \mathrm{m}, \mathrm{CH}_{2} \mathrm{CH}\right), 1.26(3 \mathrm{H}$, $\left.\mathrm{CHCH}_{3}\right) .{ }^{13} \mathrm{C} \mathrm{NMR}\left(\mathrm{CDCl}_{3}, 100 \mathrm{MHz}\right) \delta_{\mathrm{C}}: 209.2,151.9,137.8,137.7,136.1,135.0,131.6,131.2$, $128.8(\mathrm{~m}), 128.1,127.0,126.3(\mathrm{~m}), 123.9\left(\mathrm{q},{ }^{1} J \mathrm{FC}=270.8 \mathrm{~Hz}\right), 123.6,42.0,34.0,16.1$. EI-HRMS $(\mathrm{m} / \mathrm{z})$ calcd for $\mathrm{C}_{17} \mathrm{H}_{13} \mathrm{~F}_{3} \mathrm{O}\left(\mathrm{M}^{+}\right) 290.0917$, found 290.0918

2-Methyl-4-(naphthalen-2-yl)-2,3-dihydro-1H-inden-1-one $\left(\mathbf{9 a} \boldsymbol{a}_{\mathbf{2 3}}\right)$. Yield 85\%, $231 \mathrm{mg}$, colorless oil. ${ }^{1} \mathrm{H} \mathrm{NMR}\left(\mathrm{CDCl}_{3}, 400 \mathrm{MHz}\right) \delta_{\mathrm{H}}: 7.95\left(1 \mathrm{H}, \mathrm{d},{ }^{3} J_{\mathrm{HH}} 8.5 \mathrm{~Hz}, \mathrm{ArH}\right), 7.89-7.91(3 \mathrm{H}, \mathrm{m}$, ArH), $7.82\left(1 \mathrm{H}, \mathrm{d},{ }^{3} J_{\mathrm{HH}} 7.6 \mathrm{~Hz}, \mathrm{ArH}\right), 7.69-7.71(1 \mathrm{H}, \mathrm{m}, \mathrm{ArH}), 7.58-7.60(1 \mathrm{H}, \mathrm{m}, \mathrm{ArH}), 7.49-7.56$ $(3 \mathrm{H}, \mathrm{m}, \mathrm{ArH}), 3.43-3.49\left(1 \mathrm{H}, \mathrm{dd},{ }^{3} J_{\mathrm{HH}} 7.6 \mathrm{~Hz},{ }^{2} J_{\mathrm{HH}} 17.2 \mathrm{~Hz}, \mathrm{CH}_{2} \mathrm{CH}\right), 2.82\left(1 \mathrm{H}, \mathrm{dd},{ }^{3} J_{\mathrm{HH}} 4.1 \mathrm{~Hz}\right.$, $\left.{ }^{2} \mathrm{~J}_{\mathrm{HH}} 17.3 \mathrm{~Hz}, \mathrm{CH}_{2} \mathrm{CH}\right), 2.71-2.75\left(1 \mathrm{H}, \mathrm{m}, \mathrm{CH}_{2} \mathrm{CH}\right), 1.33\left(3 \mathrm{H}, \mathrm{d},{ }^{3} \mathrm{~J}_{\mathrm{HH}} 7.6 \mathrm{~Hz}, \mathrm{CHCH}_{3}\right) .{ }^{13} \mathrm{C} \mathrm{NMR}$ $\left(\mathrm{CDCl}_{3}, 100 \mathrm{MHz}\right) \delta_{\mathrm{C}}: 209.4,151.0,140.1,136.9,136.6,134.9,133.3,132.5,128.2,128.0,128.0$, $127.7,127.4,126.5,126.4,126.3,123.0,42.2,34.9,16.1$. EI-HRMS $(m / z)$ calcd for $\mathrm{C}_{20} \mathrm{H}_{16} \mathrm{O}\left(\mathrm{M}^{+}\right)$ 272.1201 , found 272.1200 . 
2-Methyl-4-(pyridin-3-yl)-2,3-dihydro-1 $\boldsymbol{H}$-inden-1-one $\left(\mathbf{9} \boldsymbol{b}_{\mathbf{1}}\right)$. Yield 73\%, $162 \mathrm{mg}$, colorless oil. ${ }^{1} \mathrm{H}$ NMR $\left(\mathrm{CDCl}_{3}, 400 \mathrm{MHz}\right) \delta_{\mathrm{H}}: 8.73(1 \mathrm{H}, \mathrm{s}, \mathrm{ArH}), 8.64\left(1 \mathrm{H}, \mathrm{d},{ }^{3} J_{\mathrm{HH}} 4.4 \mathrm{~Hz}, \mathrm{ArH}\right), 7.77-7.82(2 \mathrm{H}$, m, ArH), $7.59\left(1 \mathrm{H}, \mathrm{d},{ }^{3} J_{\mathrm{HH}} 7.3 \mathrm{~Hz}, \mathrm{ArH}\right), 7.50\left(1 \mathrm{H}, \mathrm{t},{ }^{3} J_{\mathrm{HH}} 7.5 \mathrm{~Hz}, \operatorname{ArH}\right), 7.41\left(1 \mathrm{H}, \mathrm{dd},{ }^{3} J_{\mathrm{HH}} 7.6\right.$ $\left.\mathrm{Hz},{ }^{4} J_{\mathrm{HH}} 4.8 \mathrm{~Hz}, \mathrm{ArH}\right), 3.37-3.43\left(1 \mathrm{H}, \mathrm{dd},{ }^{3} J_{\mathrm{HH}} 7.6 \mathrm{~Hz},{ }^{2} J_{\mathrm{HH}} 17.2 \mathrm{~Hz}, \mathrm{CH}_{2} \mathrm{CH}\right), 2.69-2.82(2 \mathrm{H}, \mathrm{m}$,

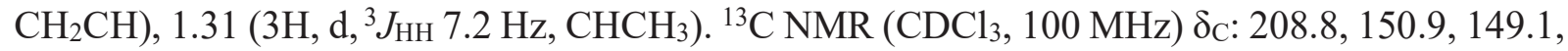
$148.7,137.1,136.5,135.7,134.8,134.7,128.2,123.7,123.4,42.1,34.6,16.0$. EI-HRMS $(\mathrm{m} / \mathrm{z})$ calcd for $\mathrm{C}_{15} \mathrm{H}_{13} \mathrm{NO}\left(\mathrm{M}^{+}\right)$223.0997, found 223.0996.

2-Methyl-4-(pyridin-4-yl)-2,3-dihydro-1 $\boldsymbol{H}$-inden-1-one $\left(\mathbf{9} \boldsymbol{b}_{\mathbf{2}}\right)$. Yield 84\%, $187 \mathrm{mg}$, white solid, mp 130-132 ${ }^{\circ} \mathrm{C} .{ }^{1} \mathrm{H} \mathrm{NMR}\left(\mathrm{CDCl}_{3}, 400 \mathrm{MHz}\right) \delta_{\mathrm{H}}: 8.68\left(1 \mathrm{H}, \mathrm{d},{ }^{3} J_{\mathrm{HH}} 5.6 \mathrm{~Hz}, \mathrm{ArH}\right), 7.80\left(1 \mathrm{H}, \mathrm{d},{ }^{3} J_{\mathrm{HH}}\right.$ $7.6 \mathrm{~Hz}, \mathrm{ArH}), 7.60\left(1 \mathrm{H}, \mathrm{d},{ }^{3} J_{\mathrm{HH}} 7.4 \mathrm{~Hz}, \mathrm{ArH}\right), 7.48\left(1 \mathrm{H}, \mathrm{t},{ }^{3} J_{\mathrm{HH}} 7.5 \mathrm{~Hz}, \mathrm{ArH}\right), 7.37\left(2 \mathrm{H}, \mathrm{d},{ }^{3} J_{\mathrm{HH}} 6.0\right.$ $\mathrm{Hz}, \mathrm{ArH}), 3.37-3.43\left(1 \mathrm{H}, \mathrm{dd},{ }^{3} J_{\mathrm{HH}} 8.0 \mathrm{~Hz},{ }^{2} J_{\mathrm{HH}} 17.2 \mathrm{~Hz}, \mathrm{CH}_{2} \mathrm{CH}\right), 2.68-2.79\left(2 \mathrm{H}, \mathrm{m}, \mathrm{CH}_{2} \mathrm{CH}\right)$, $1.29\left(3 \mathrm{H}, \mathrm{d},{ }^{3} \mathrm{~J}_{\mathrm{HH}} 7.3 \mathrm{~Hz}, \mathrm{CHCH}_{3}\right) .{ }^{13} \mathrm{C} \mathrm{NMR}\left(\mathrm{CDCl}_{3}, 100 \mathrm{MHz}\right) \delta_{\mathrm{C}}: 208.4,150.3,149.8,146.7$, 137.1, 137.0, 134.2, 128.1, 124.1, 123.1, 41.9, 34.5, 15.8. EI-HRMS $(\mathrm{m} / \mathrm{z})$ calcd for $\mathrm{C}_{15} \mathrm{H}_{13} \mathrm{NO}$ $\left(\mathrm{M}^{+}\right)$223.0997, found 223.0993.

2-Methyl-4-(pyrimidin-5-yl)-2,3-dihydro-1 $\boldsymbol{H}$-inden-1-one $\left.\mathbf{( 9}_{\mathbf{3}}\right)$. Yield 71\%, $159 \mathrm{mg}$, light yellow solid, Mp $187-189^{\circ} \mathrm{C} .{ }^{1} \mathrm{H}$ NMR $\left(\mathrm{CDCl}_{3}, 400 \mathrm{MHz}\right) \delta_{\mathrm{H}}: 9.27(1 \mathrm{H}, \mathrm{s}, \mathrm{ArH}), 8.88(2 \mathrm{H}, \mathrm{s}$, ArH), $7.88\left(1 \mathrm{H}, \mathrm{d},{ }^{3} J_{\mathrm{HH}} 7.4 \mathrm{~Hz}, \mathrm{ArH}\right), 7.62\left(1 \mathrm{H}, \mathrm{d},{ }^{3} J_{\mathrm{HH}} 6.8 \mathrm{~Hz}, \mathrm{ArH}\right), 7.55\left(1 \mathrm{H}, \mathrm{d},{ }^{3} J_{\mathrm{HH}} 7.5 \mathrm{~Hz}\right.$, ArH), 3.39-3.46 (1H, dd, $\left.{ }^{3} J_{\mathrm{HH}} 8.8 \mathrm{~Hz},{ }^{2} J_{\mathrm{HH}} 18.0 \mathrm{~Hz}, \mathrm{CH}_{2} \mathrm{CH}\right), 2.76-2.81\left(2 \mathrm{H}, \mathrm{m}, \mathrm{CH}_{2} \mathrm{CH}\right), 1.34$

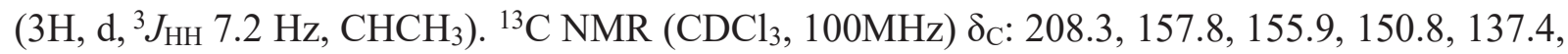
134.6, 132.91, 132.8, 128.6, 124.66, 42.1, 34.5, 16.0. EI-HRMS $(m / z)$ calcd for $\mathrm{C}_{14} \mathrm{H}_{12} \mathrm{~N}_{2} \mathrm{O}\left(\mathrm{M}^{+}\right)$ 224.0950 , found 224.0948 .

2-Methyl-4-(quinolin-3-yl)-2,3-dihydro-1 H-indan-1-one (9b $\left.\boldsymbol{b}_{\mathbf{4}}\right)$. Yield 59\%, $161 \mathrm{mg}$, colorless oil. ${ }^{1} \mathrm{H}$ NMR $\left(\mathrm{CDCl}_{3}, 400 \mathrm{MHz}\right) \delta_{\mathrm{H}}: 9.05\left(1 \mathrm{H}, \mathrm{d},{ }^{3} J_{\mathrm{HH}} 1.8 \mathrm{~Hz}, \mathrm{ArH}\right), 8.24(1 \mathrm{H}, \mathrm{s}, \mathrm{ArH}), 8.18(1 \mathrm{H}$, d, $\left.{ }^{3} J_{\mathrm{HH}} 8.5 \mathrm{~Hz}, \mathrm{ArH}\right), 7.90\left(1 \mathrm{H}, \mathrm{d},{ }^{3} J_{\mathrm{HH}} 8.0 \mathrm{~Hz}, \mathrm{ArH}\right), 7.87\left(1 \mathrm{H}, \mathrm{d},{ }^{3} J_{\mathrm{HH}} 7.6 \mathrm{~Hz}, \mathrm{ArH}\right), 7.78(1 \mathrm{H}, \mathrm{d}$, $\left.{ }^{3} J_{\mathrm{HH}} 7.6 \mathrm{~Hz}, \mathrm{ArH}\right), 7.72\left(1 \mathrm{H}, \mathrm{d},{ }^{3} J_{\mathrm{HH}} 7.2 \mathrm{~Hz}, \mathrm{ArH}\right), 7.63\left(1 \mathrm{H}, \mathrm{t},{ }^{3} J_{\mathrm{HH}} 7.6 \mathrm{~Hz}, \mathrm{ArH}\right), 7.56(1 \mathrm{H}, \mathrm{t}$, $\left.{ }^{3} J_{\mathrm{HH}} 7.6 \mathrm{~Hz}, \mathrm{ArH}\right), 3.44-3.50\left(1 \mathrm{H}, \mathrm{dd},{ }^{3} J_{\mathrm{HH}} 7.6 \mathrm{~Hz},{ }^{2} J_{\mathrm{HH}} 16.8 \mathrm{~Hz}, \mathrm{CH}_{2} \mathrm{CH}\right), 2.70-2.90(2 \mathrm{H}, \mathrm{m}$, $\left.\mathrm{CH}_{2} \mathrm{CH}\right), 1.33\left(3 \mathrm{H}, \mathrm{d},{ }^{3} \mathrm{~J}_{\mathrm{HH}} 7.2 \mathrm{~Hz}, \mathrm{CHCH}_{3}\right) .{ }^{13} \mathrm{C} \mathrm{NMR}\left(\mathrm{CDCl}_{3}, 100 \mathrm{MHz}\right) \delta_{\mathrm{C}}: 208.9,151.2,150.2$, $147.1,137.2$, 136.6, 135.2, 135.1, 132.1, 130.0, 129.1, 128.4, 127.9, 127.7, 127.3, 123.9, 42.2, 34.7, 16.1. EI-HRMS $(\mathrm{m} / \mathrm{z})$ calcd for $\left(\mathrm{M}^{+}\right) \mathrm{C}_{17} \mathrm{H}_{13} \mathrm{NO} 273.1155$, found 273.1154

2-Methyl-4-(thien-2-yl)-2,3-dihydro-1H-inden-1-one (9 $\left.\boldsymbol{b}_{5}\right)$. Yield 68\%, $155 \mathrm{mg}$, colorless oil. ${ }^{1} \mathrm{H} \mathrm{NMR}\left(\mathrm{CDCl}_{3}, 400 \mathrm{MHz}\right) \delta_{\mathrm{H}}: 7.76\left(1 \mathrm{H}, \mathrm{d},{ }^{3} J_{\mathrm{HH}} 7.6 \mathrm{~Hz}, \mathrm{ArH}\right), 7.68\left(1 \mathrm{H}, \mathrm{d},{ }^{3} J_{\mathrm{HH}} 7.5 \mathrm{~Hz}, \mathrm{ArH}\right)$, 7.36-7.40 (2H, m, ArH), $7.29\left(1 \mathrm{H}, \mathrm{d},{ }^{3} J_{\mathrm{HH}} 3.3 \mathrm{~Hz}, \mathrm{ArH}\right), 7.11-7.13(1 \mathrm{H}, \mathrm{m}, \mathrm{ArH}), 3.51-3.58(1 \mathrm{H}$, dd, $\left.{ }^{3} J_{\mathrm{HH}} 8.0 \mathrm{~Hz},{ }^{2} J_{\mathrm{HH}} 17.2 \mathrm{~Hz}, \mathrm{CH}_{2} \mathrm{CH}\right), 2.85\left(1 \mathrm{H}, \mathrm{dd},{ }^{3} J_{\mathrm{HH}} 3.9 \mathrm{~Hz},{ }^{2} J_{\mathrm{HH}} 17.2 \mathrm{~Hz}, \mathrm{CH}_{2} \mathrm{CH}\right), 2.69-$

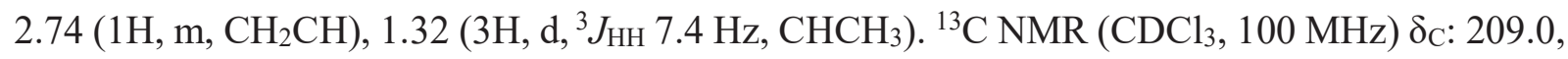
149.7, 140.8, 137.1, 133.4, 132.6, 127.9, 127.6, 125.7, 125.6, 122.8, 41.8, 35.6, 16.1. EI-HRMS $(\mathrm{m} / \mathrm{z})$ calcd for $\mathrm{C}_{14} \mathrm{H}_{12} \mathrm{OS}\left(\mathrm{M}^{+}\right) 228.0609$, found 228.0609

4-(Furan-2-yl)-2-methyl-2,3-dihydro-1H-inden-1-one (9b6). Yield 90\%, $190 \mathrm{mg}$, colorless oil. ${ }^{1} \mathrm{H} \mathrm{NMR}\left(\mathrm{CDCl}_{3}, 400 \mathrm{MHz}\right) \delta_{\mathrm{H}}: 7.93\left(1 \mathrm{H}, \mathrm{d},{ }^{3} J_{\mathrm{HH}} 7.6 \mathrm{~Hz}, \mathrm{ArH}\right), 7.64\left(1 \mathrm{H}, \mathrm{d},{ }^{3} J_{\mathrm{HH}} 7.5 \mathrm{~Hz}, \mathrm{ArH}\right)$, $7.52\left(1 \mathrm{H}, \mathrm{d},{ }^{3} J_{\mathrm{HH}} 1.5 \mathrm{~Hz}, \mathrm{ArH}\right), 7.38\left(1 \mathrm{H}, \mathrm{t},{ }^{3} J_{\mathrm{HH}} 7.6 \mathrm{~Hz}, \mathrm{ArH}\right), 6.64\left(1 \mathrm{H}, \mathrm{d},{ }^{3} J_{\mathrm{HH}} 3.4 \mathrm{~Hz}, \mathrm{ArH}\right)$, 6.50-6.52 (1H, m, ArH), 3.50-3.56 (1H, dd, $\left.{ }^{3} J_{\mathrm{HH}} 8.0 \mathrm{~Hz},{ }^{2} J_{\mathrm{HH}} 17.6 \mathrm{~Hz}, \mathrm{CH}_{2} \mathrm{C}\right), 2.87\left(1 \mathrm{H}, \mathrm{dd},{ }^{3} J_{\mathrm{HH}}\right.$ 
$\left.3.6 \mathrm{~Hz},{ }^{2} J_{\mathrm{HH}} 17.6 \mathrm{~Hz}, \mathrm{CH}_{2} \mathrm{C}\right), 2.69-2.74\left(1 \mathrm{H}, \mathrm{m}, \mathrm{CH}_{2} \mathrm{CH}\right), 1.32\left(3 \mathrm{H}, \mathrm{d},{ }^{3} J_{\mathrm{HH}} 7.8 \mathrm{~Hz}, \mathrm{CHCH}_{3}\right) .{ }^{13} \mathrm{C}$ NMR $\left(\mathrm{CDCl}_{3}, 100 \mathrm{MHz}\right) \delta_{\mathrm{C}}: 209.1,151.7,148.4,142.3,137.0,130.2,128.7,127.7,122.5,111.7$, 108.3, 41.6, 35.8, 16.2. EI-HRMS $(m / z)$ calcd for $\mathrm{C}_{14} \mathrm{H}_{12} \mathrm{O}_{2}\left(\mathrm{M}^{+}\right) 212.0837$, found 212.0831

\section{Typical procedure for 7-aryl-2-methyl-1H-indene synthesis}

To a solution of 2,3-dihydro-2-methyl-4-phenyl-1H-inden-1-one 9a $(222.3 \mathrm{mg}, 1.0 \mathrm{mmol})$ in 15 $\mathrm{mL}$ of THF/MeOH (2:1), was added $114 \mathrm{mg}\left(3.0 \mathrm{mmol}, 3.0\right.$ eq.) of $\mathrm{NaBH}_{4}$ in portions at $0{ }^{\circ} \mathrm{C}$. The reaction mixture was warmed slowly to $\mathrm{rt}$ and stirred till completion (TLC). The solvent was evaporated and $10 \mathrm{~mL}$ of water was added. The mixture was extracted with EtOAc (10 mL X 2). The combined organic extracts were dried and evaporated. The residue was taken up in $50 \mathrm{~mL}$ of toluene and mixed with $100 \mathrm{mg}$ of $\mathrm{TsOH}$ monohydrate. The formed mixture was refluxed with Dean-Stark head for 2 h. $30 \mathrm{~mL}$ of ethyl acetate was added and the resulting solution was washed with $\mathrm{Na}_{2} \mathrm{CO}_{3}(10 \%)$. The organic layer was separated and the aqueous layer was extracted with EtOAc $(30 \mathrm{~mL} \times 2)$. The combined organic extracts were dried and then filtered through a short pad of silica gel. The solvent was evaporated to give pure 2-methyl-7-phenyl-1H-indene 10a . yield: $73 \%, 151 \mathrm{mg}$, colorless oil. $1 \mathrm{H} \mathrm{NMR}\left(\mathrm{CDCl}_{3}, 400 \mathrm{MHz}\right) \delta_{\mathrm{H}}: 7.53\left(2 \mathrm{H}, \mathrm{d},{ }^{3} J_{\mathrm{HH}} 7.1 \mathrm{~Hz}\right.$, $\mathrm{ArH}), 7.44\left(2 \mathrm{H}, \mathrm{t},{ }^{3} J_{\mathrm{HH}} 7.5 \mathrm{~Hz}, \mathrm{ArH}\right), 7.35\left(\mathrm{H}, \mathrm{t},{ }^{3} J_{\mathrm{HH}} 7.4 \mathrm{~Hz}, \mathrm{ArH}\right), 7.30\left(\mathrm{H}, \mathrm{d},{ }^{3} J_{\mathrm{HH}} 7.4 \mathrm{~Hz}, \mathrm{ArH}\right)$, $7.25\left(\mathrm{H}, \mathrm{d},{ }^{3} \mathrm{~J}_{\mathrm{HH}} 5.8 \mathrm{~Hz}, \mathrm{ArH}\right), 7.13\left(1 \mathrm{H}, \mathrm{t},{ }^{3} \mathrm{~J}_{\mathrm{HH}} 7.5 \mathrm{~Hz}, \mathrm{ArH}\right), 6.54(1 \mathrm{H}, \mathrm{s}, \operatorname{ArCH}), 3.38(2 \mathrm{H}, \mathrm{s}$, $\left.\mathrm{ArCH}_{2}\right), 2.14\left(3 \mathrm{H}, \mathrm{s}, \mathrm{CCH}_{3}\right)$.

2-Methyl-7-(o-tolyl)-1H-indene (10a) $)$ Y Yield 82\%, $180 \mathrm{mg}$, colorless oil. ${ }^{1} \mathrm{H} \mathrm{NMR}\left(\mathrm{CDCl}_{3}, 400\right.$ MHz) $\delta_{\mathrm{H}}: 7.15-7.30(6 \mathrm{H}, \mathrm{m}, \mathrm{ArH}), 7.03\left(1 \mathrm{H}, \mathrm{dd},{ }^{3} J_{\mathrm{HH}} 6.7 \mathrm{~Hz},{ }^{4} J_{\mathrm{HH}} 1.8 \mathrm{~Hz}, \mathrm{ArH}\right), 6.62\left(1 \mathrm{H}, \mathrm{q},{ }^{4} J_{\mathrm{HH}}\right.$ $1.6 \mathrm{~Hz}, \mathrm{ArCH}), 3.13\left(2 \mathrm{H}, \mathrm{s}, \mathrm{ArCH}_{2}\right), 2.22\left(3 \mathrm{H}, \mathrm{s}, \mathrm{ArCH}_{3}\right), 2.17\left(3 \mathrm{H}, \mathrm{s}, \mathrm{CCH}_{3}\right)$.

2-Methyl-7-(m-tolyl)-1H-indene (10a $)$. Yield 85\%, $187 \mathrm{mg}$, colorless oil. ${ }^{1} \mathrm{HNMR}\left(\mathrm{CDCl}_{3}, 400\right.$ MHz) $\delta_{\mathrm{H}}: 7.20-7.60(7 \mathrm{H}, \mathrm{m}, \mathrm{ArH}), 6.67(1 \mathrm{H}, \mathrm{s}, \mathrm{ArCH}), 3.51\left(2 \mathrm{H}, \mathrm{s}, \mathrm{ArCH}_{2}\right), 2.55\left(3 \mathrm{H}, \mathrm{s}, \mathrm{ArCH}_{3}\right)$, $2.27\left(3 \mathrm{H}, \mathrm{s}, \mathrm{CCH}_{3}\right) .{ }^{13} \mathrm{C} \mathrm{NMR}\left(\mathrm{CDCl}_{3}, 100 \mathrm{MHz}\right) \delta_{\mathrm{C}}: 146.4,146.2,141.30,140.7,137.8,137.4$, $129.2,128.2,127.7,127.2,126.9,125.5,124.2,118.8,42.7,21.5,16.6$. EI-HRMS $(\mathrm{m} / \mathrm{z}) \mathrm{calcd}$ for $\mathrm{C}_{17} \mathrm{H}_{16}\left(\mathrm{M}^{+}\right) 220.1252$, found 220.1260

7-(2-Methoxyphenyl)-2-methyl-1H-indene (10a3). Yield 79\%, $186 \mathrm{mg}$, white solid, Mp 102 $104{ }^{\circ} \mathrm{C} .{ }^{1} \mathrm{H}$ NMR $\left(\mathrm{CDCl}_{3}, 400 \mathrm{MHz}\right) \delta_{\mathrm{H}}: 7.20-7.40(4 \mathrm{H}, \mathrm{m}, \mathrm{ArH}), 6.95-7.08$ (3H, m, ArH), 6.50 $\left(1 \mathrm{H}, \mathrm{q},{ }^{4} J_{\mathrm{HH}} 1.6 \mathrm{~Hz}, \mathrm{ArCH}\right), 3.75\left(3 \mathrm{H}, \mathrm{s}, \mathrm{OCH}_{3}\right), 3.18\left(2 \mathrm{H}, \mathrm{s}, \mathrm{ArCH}_{2}\right), 2.10\left(3 \mathrm{H}, \mathrm{s}, \mathrm{CCH}_{3}\right)$.

7-(3-Methoxyphenyl)-2-methyl-1H-indene (10a $)$ ). Yield 90\%, $212 \mathrm{mg}$, white solid, Mp 82 - 84 ${ }^{\circ} \mathrm{C} .{ }^{1} \mathrm{H}$ NMR $\left(\mathrm{CDCl}_{3}, 400 \mathrm{MHz}\right) \delta_{\mathrm{H}}: 7.20-7.38(3 \mathrm{H}, \mathrm{m}, \mathrm{ArH}), 7.02-7.15(3 \mathrm{H}, \mathrm{m}, \mathrm{ArH}), 6.88(1 \mathrm{H}$, dd, $\left.{ }^{3} J_{\mathrm{HH}} 8.4 \mathrm{~Hz},{ }^{4} \mathrm{JHH} 2.0 \mathrm{~Hz}, \mathrm{ArH}\right), 6.60\left(1 \mathrm{H}, \mathrm{q},{ }^{4} J_{\mathrm{HH}} 1.2 \mathrm{~Hz}, \mathrm{ArCH}\right), 3.91\left(3 \mathrm{H}, \mathrm{s}, \mathrm{OCH}_{3}\right), 3.45$

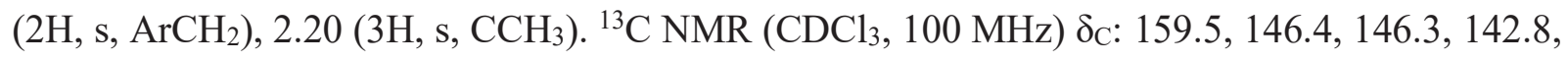
140.7, 137.2, 129.3, 127.1, 126.9, 124.1, 120.9, 118.9, 114.2, 112.4, 55.2, 42.7, 16.6. EI-HRMS $(\mathrm{m} / \mathrm{z})$ calcd for $\mathrm{C}_{17} \mathrm{H}_{16} \mathrm{O}\left(\mathrm{M}^{+}\right)$236.1201, found 236.1210.

7-(4-Methoxyphenyl)-2-methyl-1H-indene (10 $\left.a_{5}\right)$. Yield 95\%, $224 \mathrm{mg}$, white solid, Mp 85 - 87 ${ }^{\circ} \mathrm{C} .{ }^{1} \mathrm{H}$ NMR $\left(\mathrm{CDCl}_{3}, 400 \mathrm{MHz}\right) \delta_{\mathrm{H}}:$ 7.43-7.46 $(2 \mathrm{H}, \mathrm{m}, \mathrm{ArH}), 7.27-7.31(1 \mathrm{H}, \mathrm{m}, \mathrm{ArH}), 7.20-7.22$ $(1 \mathrm{H}, \mathrm{m}, \mathrm{ArH}), 7.09-7.12(1 \mathrm{H}, \mathrm{m}, \mathrm{ArH}), 7.94-7.97(2 \mathrm{H}, \mathrm{m}, \mathrm{ArH}), 6.51(1 \mathrm{H}, \mathrm{s}, \mathrm{ArCH}), 3.81$ (3H, s,

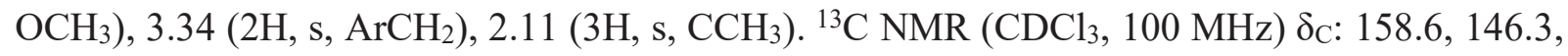


146.1, 140.6, 136.9, 133.7, 129.4, 127.1, 126.9, 124.1, 118.5, 113.7, 55.1, 42.7, 16.6. EI-HRMS $(\mathrm{m} / \mathrm{z})$ calcd for $\mathrm{C}_{17} \mathrm{H}_{16} \mathrm{O}\left(\mathrm{M}^{+}\right) 236.1201$, found 236.1204 .

7-(3,5-Dimethylphenyl)-2-methyl-1H-indene (10 $\left.\boldsymbol{a}_{\mathbf{6}}\right)$. Yield 93\%, $217 \mathrm{mg}$, white solid, Mp 57 $59{ }^{\circ} \mathrm{C} .{ }^{1} \mathrm{HNMR}\left(\mathrm{CDCl}_{3}, 400 \mathrm{MHz}\right) \delta_{\mathrm{H}}: 7.25-7.50(5 \mathrm{H}, \mathrm{m}, \mathrm{ArH}), 7.16(1 \mathrm{H}, \mathrm{s}, \mathrm{ArH}), 6.69(1 \mathrm{H}, \mathrm{s}$, $\mathrm{ArCH}), 3.54\left(2 \mathrm{H}, \mathrm{s}, \mathrm{ArCH}_{2}\right), 2.55\left(6 \mathrm{H}, \mathrm{s}, \mathrm{ArCH}_{3}\right), 2.29\left(3 \mathrm{H}, \mathrm{s}, \mathrm{CCH}_{3}\right) .{ }^{13} \mathrm{C} \mathrm{NMR}\left(\mathrm{CDCl}_{3}, 100\right.$ MHz) $\delta_{\mathrm{C}}: 146.3,146.1,141.3,140.7,137.7,137.5,128.6,127.2,126.8,126.3,124.2,118.7,42.7$, 21.4, 16.6. EI-HRMS $(\mathrm{m} / \mathrm{z})$ calcd for $\mathrm{C}_{18} \mathrm{H}_{18}\left(\mathrm{M}^{+}\right) 234.1409$, found 234.1415

7-(4-(tert-Butyl)phenyl)-2-methyl-1H-indene (10 $\left.\boldsymbol{~}_{7}\right)$. Yield 94\%, $246 \mathrm{mg}$, white solid, Mp 66 $68{ }^{\circ} \mathrm{C} .{ }^{1} \mathrm{H}$ NMR $\left(\mathrm{CDCl}_{3}, 400 \mathrm{MHz}\right) \delta_{\mathrm{H}}: 7.51-7.54(4 \mathrm{H}, \mathrm{m}, \mathrm{ArH}), 7.15-7.40(3 \mathrm{H}, \mathrm{m}, \mathrm{ArH}), 6.60(1 \mathrm{H}$, $\mathrm{s}, \mathrm{ArCH}), 3.47\left(2 \mathrm{H}, \mathrm{s}, \mathrm{ArCH}_{2}\right), 2.20\left(3 \mathrm{H}, \mathrm{s}, \mathrm{CCH}_{3}\right), 1.45(9 \mathrm{H}, \mathrm{s}, t \mathrm{Bu}) \cdot{ }^{13} \mathrm{C} \mathrm{NMR}\left(\mathrm{CDCl}_{3}, 100 \mathrm{MHz}\right)$ $\delta_{\mathrm{C}}: 149.9,146.5,146.3,140.9,138.4,137.3,128.2,127.2,127.0,125.4,124.3,118.8,42.9,34.6$, 31.5, 16.8. EI-HRMS $(\mathrm{m} / z)$ calcd for $\mathrm{C}_{20} \mathrm{H}_{22}\left(\mathrm{M}^{+}\right) 262.1722$, found 262.1713

2-Methyl-7-(4-(trifluoromethoxy)phenyl)-1H-indene (10a8). Yield 96\%, $278 \mathrm{mg}$, colorless oil. ${ }^{1} \mathrm{HNMR}\left(\mathrm{CDCl}_{3}, 400 \mathrm{MHz}\right) \delta_{\mathrm{H}}: 7.59-7.63(2 \mathrm{H}, \mathrm{m}, \mathrm{ArH}), 7.18-7.30(4 \mathrm{H}, \mathrm{m}, \mathrm{ArH}), 7.03(1 \mathrm{H}, \mathrm{dd}$, $\left.{ }^{3} J_{\mathrm{HH}} 7.2 \mathrm{~Hz},{ }^{4} J_{\mathrm{HH}} 1.2 \mathrm{~Hz}, \mathrm{ArH}\right), 6.65-6.66\left(1 \mathrm{H}, \mathrm{q},{ }^{4} J_{\mathrm{HH}} 1.6 \mathrm{~Hz}, \mathrm{ArCH}\right), 3.43\left(2 \mathrm{H}, \mathrm{s}, \mathrm{ArCH}_{2}\right), 2.25$ $\left(3 \mathrm{H}, \mathrm{s}, \mathrm{CCH}_{3}\right) .{ }^{13} \mathrm{C} \mathrm{NMR}(\mathrm{CDCl} 3,100 \mathrm{MHz}) \delta \mathrm{C}: 148.3,146.6,146.4,140.7,140.0,135.9,129.7$, 127.2, 127.1, 124.1, 120.8, 120.7 (q, $\left.{ }^{1} J \mathrm{FC} 255.5 \mathrm{~Hz}\right), 119.3,42.6,29.8,16.5$. EI-HRMS $(\mathrm{m} / \mathrm{z})$ calcd for $\mathrm{C}_{17} \mathrm{H}_{13} \mathrm{~F}_{3} \mathrm{O}\left(\mathrm{M}^{+}\right) 290.0918$, found 290.0923

7-(2-Fluorophenyl)-2-methyl-1H-indene (10a9). Yield 97\%, $217 \mathrm{mg}$, white solid, Mp 56-58 ${ }^{\circ} \mathrm{C}$.

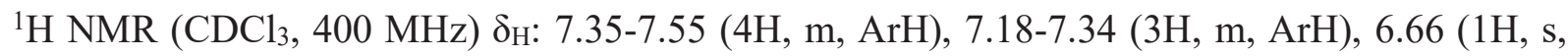
$\mathrm{ArCH}), 3.38\left(2 \mathrm{H}, \mathrm{s}, \mathrm{ArCH}_{2}\right), 2.24\left(3 \mathrm{H}, \mathrm{s}, \mathrm{CCH}_{3}\right)$.

7-(3-Fluorophenyl)-2-methyl-1H-indene (10a10). Yield 96\%, $215 \mathrm{mg}$, colorless oil. ${ }^{1} \mathrm{H}$ NMR $\left(\mathrm{CDCl}_{3}, 400 \mathrm{MHz}\right) \delta_{\mathrm{H}}: 7.43-7.48(1 \mathrm{H}, \mathrm{m}, \mathrm{ArH}), 7.30-7.38(4 \mathrm{H}, \mathrm{m}, \mathrm{ArH}), 7.19\left(1 \mathrm{H},{ }^{3} \mathrm{~J}_{\mathrm{HH}} 7.2 \mathrm{~Hz}\right.$, $\mathrm{ArH}), 7.09-7.14(1 \mathrm{H}, \mathrm{m}, \mathrm{ArH}), 6.61(1 \mathrm{H}, \mathrm{s}, \mathrm{ArCH}), 3.43\left(2 \mathrm{H}, \mathrm{s}, \mathrm{ArCH}_{2}\right), 2.21\left(3 \mathrm{H}, \mathrm{s}, \mathrm{CCH}_{3}\right) .{ }^{13} \mathrm{C}$ NMR $\left(\mathrm{CDCl}_{3}, 100 \mathrm{MHz}\right) \delta_{\mathrm{C}}: 162.8\left(\mathrm{~d},{ }^{1} J \mathrm{FC} 244.1 \mathrm{~Hz}\right), 146.6,146.4,143.6\left(\mathrm{~d},{ }^{3} J \mathrm{FC} 7.6 \mathrm{~Hz}\right), 143.5$, 140.7, 136.0, 129.8 (d, $\left.{ }^{3} J \mathrm{FC} 8.4 \mathrm{~Hz}\right), 127.1$ (d, $\left.{ }^{4} J \mathrm{FC} 5.4 \mathrm{~Hz}\right), 124.1\left(\mathrm{~d},{ }^{4} J \mathrm{FC} 2.7 \mathrm{~Hz}\right), 124.0,119.4$, $115.3\left(\mathrm{~d},{ }^{2} \mathrm{JFC} 20.4 \mathrm{~Hz}\right), 113.9$ (d, $\left.{ }^{2} \mathrm{FC} 21.0 \mathrm{~Hz}\right), 42.6,16.6$. EI-HRMS $(\mathrm{m} / \mathrm{z})$ calcd for $\mathrm{C}_{16} \mathrm{H}_{13} \mathrm{~F}$ $\left(\mathrm{M}^{+}\right)$224.1001, found 224.0998.

7-(4-Fluorophenyl)-2-methyl- $\mathbf{H}$-indene $\left(\mathbf{1 0} \boldsymbol{a}_{11}\right.$, contains indene double bond isomers (1:1.2)). Yield 90\%, $201 \mathrm{mg}$, white solid. ${ }^{1} \mathrm{H} \mathrm{NMR}\left(\mathrm{CDCl}_{3}, 400 \mathrm{MHz}\right) \delta_{\mathrm{H}}: 7.40-7.55(2 \mathrm{H}, \mathrm{ArH})$, 7.05-7.39 (5H, ArH), 6.61 (0.55H, s ArCH), 6.53 (0.45H, m ArCH) $3.36\left(1.1 \mathrm{H}, \mathrm{s}, \mathrm{ArCH}_{2}\right), 3.35$ $\left(0.9 \mathrm{H}, \mathrm{s}, \mathrm{ArCH}_{2}\right), 2.14\left(3 \mathrm{H}, \mathrm{CCH}_{3}\right) .{ }^{13} \mathrm{C} \mathrm{NMR}\left(\mathrm{CDCl}_{3}, 100 \mathrm{MHz}\right) \delta_{\mathrm{C}}: 162.0\left(\mathrm{~d},{ }^{1} J \mathrm{FC} 241.8 \mathrm{~Hz}\right)$, 146.9, 146.5 (d, $\left.{ }^{3} J \mathrm{FC} 11.2 \mathrm{~Hz}\right), 144.0,143.5,140.8,136.3,132.8,130.2\left(\mathrm{~d},{ }^{3} J \mathrm{FC} 7.8 \mathrm{~Hz}\right), 130.0$ (d, $\left.{ }^{3} J \mathrm{FC} 7.9 \mathrm{~Hz}\right), 127.1$ (d, $\left.{ }^{3} J \mathrm{FC} 11.2 \mathrm{~Hz}\right), 126.6,125.9,124.1,123.9,122.4,119.0,115.2\left(\mathrm{~d},{ }^{2} J \mathrm{FC}\right.$ $21.1 \mathrm{~Hz}), 115.1$ (d, $\left.{ }^{2} \mathrm{FC} 21.2 \mathrm{~Hz}\right), 42.9$, 16.9. EI-HRMS ( $/ \mathrm{z}$ ) calcd for $\mathrm{C}_{16} \mathrm{H}_{13} \mathrm{~F}\left(\mathrm{M}^{+}\right) 224.1001$, found 224.1009.

7-(4-Chlorophenyl)-2-methyl-1H-indene (10a $a_{12}$ contains indene double bond isomers (1:1.4)). Yield 77\%, $184 \mathrm{mg}$, colorless oil. ${ }^{1} \mathrm{H}$ NMR $\left(\mathrm{CDCl}_{3}, 400 \mathrm{MHz}\right) \delta_{\mathrm{H}}: 7.17-7.53(6.6 \mathrm{H}, \mathrm{m}$, $\operatorname{ArH}), 7.13\left(0.41 \mathrm{H}, \mathrm{dd},{ }^{3} J_{\mathrm{HH}} 7.6 \mathrm{~Hz},{ }^{4} J_{\mathrm{HH}} 1.2 \mathrm{~Hz}, \operatorname{ArH}\right), 6.61(0.59 \mathrm{H}, \mathrm{s}, \operatorname{ArCH}), 6.53(0.41 \mathrm{H}, \mathrm{q}$, $\left.{ }^{4} J_{\mathrm{HH}} 1.6 \mathrm{~Hz}, \mathrm{ArCH}\right), 3.35\left(1.22 \mathrm{H}, \mathrm{s}, \mathrm{ArCH}_{2}\right), 3.32\left(0.87 \mathrm{H}, \mathrm{s}, \mathrm{ArCH}_{2}\right), 2.14\left(1.79 \mathrm{H}, \mathrm{CCH}_{3}\right), 2.13$ 
$\left(1.29 \mathrm{H}, \mathrm{CCH}_{3}\right) .{ }^{13} \mathrm{C} \mathrm{NMR}\left(\mathrm{CDCl}_{3}, 100 \mathrm{MHz}\right) \delta_{\mathrm{C}}: 147.0,144.1,143.5,139.6,130.1,128.5,127.2$, 126.5, 125.9, 123.9, 122.6, 119.2, 42.9, 16.8. EI-HRMS $(\mathrm{m} / \mathrm{z})$ calcd for $\mathrm{C}_{16} \mathrm{H}_{13} \mathrm{Cl}\left(\mathrm{M}^{+}\right) 240.0706$, found 240.0715

2-Methyl-7-(3-(trifluoromethyl)phenyl)-1H-indene (10 $\left.\boldsymbol{a}_{\mathbf{1 3}}\right)$. Yield 90\%, $246 \mathrm{mg}$, colorless oil. ${ }^{1} \mathrm{H}$ NMR $\left(\mathrm{CDCl}_{3}, 400 \mathrm{MHz}\right) \delta_{\mathrm{H}}: 7.86(1 \mathrm{H}, \mathrm{s} \mathrm{ArH}), 7.75\left(1 \mathrm{H}, \mathrm{d},{ }^{3} J_{\mathrm{HH}} 7.6 \mathrm{~Hz}, \operatorname{ArH}\right), 7.67(1 \mathrm{H}, \mathrm{d}$, $\left.{ }^{3} J_{\mathrm{HH}} 7.8 \mathrm{~Hz}, \mathrm{ArH}\right), 7.59\left(1 \mathrm{H}, \mathrm{t},{ }^{3} J_{\mathrm{HH}} 7.7 \mathrm{~Hz}, \mathrm{ArH}\right), 7.39\left(1 \mathrm{H}, \mathrm{t},{ }^{3} J_{\mathrm{HH}} 7.4 \mathrm{~Hz}, \mathrm{ArH}\right), 7.33(1 \mathrm{H}, \mathrm{d}$, $\left.{ }^{3} J_{\mathrm{HH}} 7.3 \mathrm{~Hz}, \mathrm{ArH}\right), 7.17\left(1 \mathrm{H}, \mathrm{d},{ }^{3} J_{\mathrm{HH}} 7.3 \mathrm{~Hz}, \mathrm{ArH}\right), 6.61(1 \mathrm{H}, \mathrm{s} \mathrm{ArCH}), 3.40\left(2 \mathrm{H}, \mathrm{s}, \mathrm{ArCH}_{2}\right), 2.20$ $\left(3 \mathrm{H}, \mathrm{CCH}_{3}\right) .{ }^{13} \mathrm{C} \mathrm{NMR}\left(\mathrm{CDCl}_{3}, 100 \mathrm{MHz}\right) \delta_{\mathrm{C}}: 146.7,146.4,142.1,140.8,135.9,131.7,130.8(\mathrm{q}$, $\left.{ }^{2} J \mathrm{FC} 31.9 \mathrm{~Hz}\right), 128.8,127.2,127.2,125.3$ (q, $\left.{ }^{4} J \mathrm{FC} 3.7 \mathrm{~Hz}\right), 124.4$ (q, $\left.{ }^{1} J \mathrm{FC} 270.5 \mathrm{~Hz}\right), 124.1,123.8$ (q, $\left.{ }^{4} \mathrm{JFC} 3.7 \mathrm{~Hz}\right), 119.6,42.5$, 16.6. EI-HRMS $(\mathrm{m} / \mathrm{z})$ calcd for $\mathrm{C}_{17} \mathrm{H}_{13} \mathrm{~F}_{3}\left(\mathrm{M}^{+}\right) 274.0969$, found 274.0978 .

2-Methyl-7-(4-(trifluoromethyl)phenyl)- $\mathbf{H}$-indene ( $10 \boldsymbol{a}_{14}$ contains indene double bond isomers (1:3.6)). Yield 97\%, $265 \mathrm{mg}$, white solid, Mp 145-147 ${ }^{\circ} \mathrm{C} .{ }^{1} \mathrm{H}$ NMR $\left(\mathrm{CDCl}_{3}, 400 \mathrm{MHz}\right) \delta_{\mathrm{H}}: 7.68$ $\left(2 \mathrm{H}, \mathrm{d},{ }^{3} J_{\mathrm{HH}} 8.0 \mathrm{~Hz}, \mathrm{ArH}\right), 7.62\left(2.40 \mathrm{H}, \mathrm{d},{ }^{3} J_{\mathrm{HH}} 8.4 \mathrm{~Hz}, \mathrm{ArH}\right), 7.10\left(0.78 \mathrm{H}, \mathrm{d},{ }^{3} J_{\mathrm{HH}} 7.6 \mathrm{~Hz}, \mathrm{ArH}\right)$, $6.62(0.22 \mathrm{H}, \mathrm{s}, \mathrm{ArCH}), 6.54(0.78 \mathrm{H}, \mathrm{s}, \mathrm{ArCH}), 3.37\left(0.48 \mathrm{H}, \mathrm{s}, \mathrm{ArCH}_{2}\right), 3.34\left(1.61 \mathrm{H}, \mathrm{s}, \mathrm{ArCH}_{2}\right)$, $2.14\left(3 \mathrm{H}, \mathrm{CCH}_{3}\right) .{ }^{13} \mathrm{C} \mathrm{NMR}\left(\mathrm{CDCl}_{3}, 100 \mathrm{MHz}\right) \delta_{\mathrm{C}}: 146.7,146.5,140.8,135.9,129.2\left(\mathrm{q},{ }^{2} J \mathrm{FC} 32.7\right.$ Hz) 129.1, 128.7, 127.2, 127.2, 125.3 (q, $\left.{ }^{4} J \mathrm{FC} 3.8 \mathrm{~Hz}\right), 124.4$ (q, $\left.{ }^{1} J \mathrm{FC} 266.1 \mathrm{~Hz}\right), 124.1,119.6$, 42.6, 16.7. EI-HRMS $(\mathrm{m} / z)$ calcd for $\mathrm{C}_{17} \mathrm{H}_{13} \mathrm{~F}_{3}\left(\mathrm{M}^{+}\right)$274.0969, found 274.0979.

7-(3,5-Bis(trifluoromethyl)phenyl)-2-methyl-1H-indene (10a15). Yield 87\%, $297 \mathrm{mg}$, white solid, Mp 65-67 ${ }^{\circ} \mathrm{C} .{ }^{1} \mathrm{H}$ NMR $\left(\mathrm{CDCl}_{3}, 400 \mathrm{MHz}\right) \delta_{\mathrm{H}}: 8.01(2 \mathrm{H}, \mathrm{s} \mathrm{ArH}), 7.91(1 \mathrm{H}, \mathrm{s} \mathrm{ArH}), 7.33-$ $7.40(2 \mathrm{H}, \mathrm{m} \mathrm{ArH}), 7.14\left(1 \mathrm{H}, \mathrm{d},{ }^{3} J_{\mathrm{HH}} 7.2 \mathrm{~Hz}, \mathrm{ArH}\right), 6.59(1 \mathrm{H}, \mathrm{s}, \mathrm{ArCH}), 3.35\left(2 \mathrm{H}, \mathrm{s}, \mathrm{ArCH}_{2}\right), 2.19$ $\left(3 \mathrm{H}, \mathrm{CCH}_{3}\right) .{ }^{13} \mathrm{C} \mathrm{NMR}\left(\mathrm{CDCl}_{3}, 100 \mathrm{MHz}\right) \delta_{\mathrm{C}}: 147.0,146.6,143.5,140.8,134.4,131.8\left(\mathrm{q},{ }^{2} J \mathrm{FC}\right.$ $27.1 \mathrm{~Hz}), 128.5$ (m), 127.5, 127.3, 123.9, 123.4 (q, ${ }^{1} J \mathrm{FC} 271.2 \mathrm{~Hz}$ ), 120.8 (m), 120.3, 42.3, 16.6. EI-HRMS $(\mathrm{m} / \mathrm{z})$ calcd for $\mathrm{C}_{18} \mathrm{H}_{12} \mathrm{~F}_{6}\left(\mathrm{M}^{+}\right) 342.0843$, found 342.0835 .

3-(2-Methyl-1H-inden-7-yl)benzonitrile (10a16). Yield 93\%, $214 \mathrm{mg}$, white solid, Mp $91-93{ }^{\circ} \mathrm{C}$. ${ }^{1} \mathrm{H}$ NMR $\left(\mathrm{CDCl}_{3}, 400 \mathrm{MHz}\right) \delta_{\mathrm{H}}: 7.82\left(1 \mathrm{H}, \mathrm{t},{ }^{4} J_{\mathrm{HH}} 1.4 \mathrm{~Hz}, \mathrm{ArH}\right), 7.75\left(1 \mathrm{H}, \mathrm{dt},{ }^{3} J_{\mathrm{HH}} 8.4 \mathrm{~Hz},{ }^{4} J_{\mathrm{HH}}\right.$ $1.6 \mathrm{~Hz}, \mathrm{ArH}), 7.65\left(1 \mathrm{H}, \mathrm{dt},{ }^{3} J_{\mathrm{HH}} 7.6 \mathrm{~Hz},{ }^{4} J_{\mathrm{HH}} 1.6 \mathrm{~Hz}, \mathrm{ArH}\right), 7.55\left(1 \mathrm{H}, \mathrm{t},{ }^{4} J_{\mathrm{HH}} 7.9 \mathrm{~Hz}, \mathrm{ArH}\right), 7.29-$ $7.36(2 \mathrm{H}, \mathrm{m} \mathrm{ArH}), 7.09\left(1 \mathrm{H}, \mathrm{dd},{ }^{3} J_{\mathrm{HH}} 7.2 \mathrm{~Hz},{ }^{4} J_{\mathrm{HH}} 1.4 \mathrm{~Hz}, \mathrm{ArH}\right), 6.56\left(1 \mathrm{H}, \mathrm{q},{ }^{4} J_{\mathrm{HH}} 1.6 \mathrm{~Hz}, \mathrm{ArCH}\right)$, $3.33\left(2 \mathrm{H}, \mathrm{s}, \mathrm{ArCH}_{2}\right), 2.17\left(3 \mathrm{H}, \mathrm{CCH}_{3}\right) .{ }^{13} \mathrm{C} \mathrm{NMR}\left(\mathrm{CDCl}_{3}, 100 \mathrm{MHz}\right) \delta_{\mathrm{C}}: 146.7,146.4,142.4,140.6$, 134.8, 132.7, 131.7, 130.5, 129.2, 127.3, 127.1, 123.8, 119.8, 118.8, 42.4, 16.6. EI-HRMS $(\mathrm{m} / \mathrm{z})$ calcd for $\mathrm{C}_{17} \mathrm{H}_{13} \mathrm{~N}\left(\mathrm{M}^{+}\right) 231.1048$, found 231.1053 .

4-(2-Methyl-1H-inden-7-yl)benzonitrile ( $10 a_{17}$ contains indene double bond isomers (1:3.3)). Yield 75\%, $173 \mathrm{mg}$, white solid. (Major) ${ }^{1} \mathrm{H} \mathrm{NMR}\left(\mathrm{CDCl}_{3}, 400 \mathrm{MHz}\right) \delta_{\mathrm{H}}: 7.73\left(2 \mathrm{H}, \mathrm{d},{ }^{3} J_{\mathrm{HH}} 8.3\right.$ $\mathrm{Hz}, \mathrm{ArH}), 7.62\left(2 \mathrm{H}, \mathrm{d},{ }^{3} J_{\mathrm{HH}} 7.2 \mathrm{~Hz}, \mathrm{ArH}\right), 7.15-7.45(2 \mathrm{H}, \mathrm{m}, \mathrm{ArH}), 7.10\left(1 \mathrm{H}, \mathrm{dd},{ }^{3} J_{\mathrm{HH}} 7.2 \mathrm{~Hz},{ }^{4} J_{\mathrm{HH}}\right.$ $1.2 \mathrm{~Hz}, \mathrm{ArH}), 6.55(1 \mathrm{H}, \mathrm{q}, 1.6 \mathrm{~Hz}, \mathrm{ArCH}), 3.35\left(2 \mathrm{H}, \mathrm{s}, \mathrm{ArCH}_{2}\right), 2.16\left(3 \mathrm{H}, \mathrm{CCH}_{3}\right) .{ }^{13} \mathrm{C} \mathrm{NMR}$ $\left(\mathrm{CDCl}_{3}, 100 \mathrm{MHz}\right) \delta_{\mathrm{C}}: 146.8,146.5,146.1,140.7,135.4,132.2,129.4,129.1,127.3,127.1,123.9$, 120.0, 110.7, 42.6, 16.6. EI-HRMS $(\mathrm{m} / \mathrm{z})$ calcd for $\mathrm{C}_{17} \mathrm{H}_{13} \mathrm{~N}\left(\mathrm{M}^{+}\right)$231.1048, found 231.1053.

7-([1,1'-Biphenyl]-4-yl)-2-methyl-1H-indene (10a18). Yield 91\%, 256 mg, white solid, Mp 138$140{ }^{\circ} \mathrm{C} .{ }^{1} \mathrm{H} \mathrm{NMR}\left(\mathrm{CDCl}_{3}, 400 \mathrm{MHz}\right) \delta_{\mathrm{H}}: 7.65(5 \mathrm{H}, \mathrm{m}, \mathrm{ArH}), 7.47\left(2 \mathrm{H}, \mathrm{t},{ }^{3} J_{\mathrm{HH}} 7.6 \mathrm{~Hz}, \mathrm{ArH}\right), 7.25-$ $7.40(3 \mathrm{H}, \mathrm{m}, \mathrm{ArH}), 7.19\left(1 \mathrm{H}, \mathrm{d},{ }^{3} J_{\mathrm{HH}} 7.2 \mathrm{~Hz}, \mathrm{ArH}\right), 6.56(1 \mathrm{H}, \mathrm{s}, \mathrm{ArCH}), 3.44\left(2 \mathrm{H}, \mathrm{s}, \mathrm{ArCH}_{2}\right), 2.16$ 
$\left(3 \mathrm{H}, \mathrm{CCH}_{3}\right) .{ }^{13} \mathrm{C} \mathrm{NMR}\left(\mathrm{CDCl}_{3}, 100 \mathrm{MHz}\right) \delta_{\mathrm{C}}: 146.5,146.3,140.8,140.8,140.3,139.8,136.9$, $128.8,128.8,127.3,127.2,127.1,127.0,124.2,119.0,42.8,16.7$ EI-HRMS $(\mathrm{m} / z)$ calcd for $\mathrm{C}_{22} \mathrm{H}_{18}$ $\left(\mathrm{M}^{+}\right)$282.1409, found 282.1419.

7-(3-Chlorophenyl)-2-methyl-1H-indene (10a $\mathbf{1 0})$. Yield 89\%, $213 \mathrm{mg}$, colorless oil. ${ }^{1} \mathrm{H}$ NMR $\left(\mathrm{CDCl}_{3}, 400 \mathrm{MHz}\right) \delta_{\mathrm{H}}: 7.46(1 \mathrm{H}, \mathrm{s}, \mathrm{ArH}), 7.16-7.35(5 \mathrm{H}, \mathrm{m}, \mathrm{ArH}), 7.04\left(1 \mathrm{H}, \mathrm{dd},{ }^{3} J_{\mathrm{HH}} 7.4 \mathrm{~Hz},{ }^{4} J_{\mathrm{HH}}\right.$ $1.2 \mathrm{~Hz}, \mathrm{ArH}), 6.47-6.49\left(1 \mathrm{H}, \mathrm{q},{ }^{4} J_{\mathrm{HH}} 1.6 \mathrm{~Hz}, \mathrm{ArCH}\right), 3.29\left(2 \mathrm{H}, \mathrm{s}, \mathrm{ArCH}_{2}\right), 2.09\left(3 \mathrm{H}, \mathrm{CCH}_{3}\right) .{ }^{13} \mathrm{C}$ NMR $\left(\mathrm{CDCl}_{3}, 100 \mathrm{MHz}\right) \delta_{\mathrm{C}}: 146.6,146.4,143.1,140.7,135.9,134.2,129.6,128.5,127.1,127.1$, 127.0, 126.6, 124.0, 119.4, 42.6, 16.6. EI-HRMS $(\mathrm{m} / \mathrm{z})$ calcd for $\mathrm{C}_{16} \mathrm{H}_{13} \mathrm{Cl}\left(\mathrm{M}^{+}\right)$240.0706, found 240.0717 .

2-(2-Methyl-1H-inden-7-yl)naphthalene (10a $\left.\boldsymbol{a}_{23}\right)$. Yield 94\%, $240 \mathrm{mg}$, light yellow solid, Mp 81 - $83{ }^{\circ} \mathrm{C} .{ }^{1} \mathrm{H} \mathrm{NMR}\left(\mathrm{CDCl}_{3}, 400 \mathrm{MHz}\right) \delta_{\mathrm{H}}: 8.01(1 \mathrm{H}, \mathrm{s}, \mathrm{ArH}), 7.90-7.95(3 \mathrm{H}, \mathrm{m}, \mathrm{ArH}), 7.71(1 \mathrm{H}, \mathrm{dd}$, $\left.{ }^{3} J_{\mathrm{HH}} 8.4 \mathrm{~Hz},{ }^{4} J_{\mathrm{HH}} 1.8 \mathrm{~Hz}, \mathrm{ArH}\right), 7.51-7.56(2 \mathrm{H}, \mathrm{m}, \mathrm{ArH}), 7.39\left(1 \mathrm{H}, \mathrm{t},{ }^{3} J_{\mathrm{HH}} 7.5 \mathrm{~Hz}, \mathrm{ArH}\right), 7.26-7.34$ $(2 \mathrm{H}, \mathrm{m}, \mathrm{ArH}), 6.60\left(1 \mathrm{H}, \mathrm{q},{ }^{4} \mathrm{JHH}_{\mathrm{HH}} .6 \mathrm{~Hz}, \mathrm{ArCH}\right), 3.46\left(2 \mathrm{H}, \mathrm{s}, \mathrm{ArCH}_{2}\right), 2.18\left(3 \mathrm{H}, \mathrm{CCH}_{3}\right) .{ }^{13} \mathrm{C} \mathrm{NMR}$ $\left(\mathrm{CDCl}_{3}, 100 \mathrm{MHz}\right) \delta_{\mathrm{C}}: 146.5,146.4,141.0,138.8,137.3,133.4,132.4,128.0,127.9,127.7,127.2$, 127.1, 127.1, 126.9, 126.2, 125.9, 124.5, 119.0, 42.8, 16.7. EI-HRMS $(\mathrm{m} / \mathrm{z})$ calcd for $\mathrm{C}_{20} \mathrm{H}_{16}\left(\mathrm{M}^{+}\right)$, 256.1252, found 256.1258

3-(2-Methyl-1H-inden-7-yl)pyridine (10 $\boldsymbol{b}_{\mathbf{1}}$ contains indene double bond isomers (1:5)). Yield 89\%, $184 \mathrm{mg}$, colorless oil. (Major) ${ }^{1} \mathrm{HNMR}\left(\mathrm{CDCl}_{3}, 400 \mathrm{MHz}\right) \delta_{\mathrm{H}}: 8.77(1 \mathrm{H}, \mathrm{s}, \mathrm{ArH}), 8.56(1 \mathrm{H}$, d, $\left.{ }^{3} J_{\mathrm{HH}} 8.0 \mathrm{~Hz}, \mathrm{ArH}\right), 7.81\left(1 \mathrm{H}, \mathrm{dd},{ }^{3} J_{\mathrm{HH}} 7.6 \mathrm{~Hz},{ }^{4} J_{\mathrm{HH}} 1.8 \mathrm{~Hz}, \mathrm{ArH}\right), 7.15-7.40(3 \mathrm{H}, \mathrm{m}, \mathrm{ArH}), 7.09$ $\left(1 \mathrm{H}, \mathrm{dd},{ }^{3} J_{\mathrm{HH}} 7.2 \mathrm{~Hz},{ }^{4} J_{\mathrm{HH}} 0.8 \mathrm{~Hz}, \mathrm{ArH}\right), 6.53\left(1 \mathrm{H}, \mathrm{q},{ }^{4} J_{\mathrm{HH}} 1.6 \mathrm{~Hz}, \mathrm{ArCH}\right), 3.33\left(2 \mathrm{H}, \mathrm{s}, \mathrm{ArCH}_{2}\right)$,

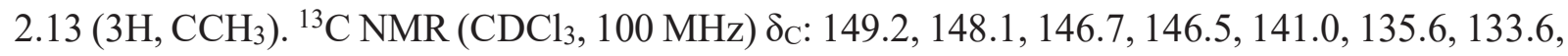
127.2, 127.1, 124.0, 123.3, 119.6, 42.5, 16.6. EI-HRMS $(\mathrm{m} / \mathrm{z})$ calcd for $\mathrm{C}_{15} \mathrm{H}_{13} \mathrm{~N}\left(\mathrm{M}^{+}\right)$207.1048, found 207.1057.

4-(2-Methyl-1H-inden-7-yl)pyridine (10b $)$. Yield 98\%, $202 \mathrm{mg}$, colorless oil. ${ }^{1} \mathrm{H} \mathrm{NMR}\left(\mathrm{CDCl}_{3}\right.$, $400 \mathrm{MHz}) \delta_{\mathrm{H}}: 8.66\left(2 \mathrm{H}, \mathrm{d},{ }^{3} J_{\mathrm{HH}} 6.0 \mathrm{~Hz}, \mathrm{ArH}\right), 7.43-7.45(2 \mathrm{H}, \mathrm{m}, \mathrm{ArH}), 7.29-7.36$ (2H, m, ArH), $7.13\left(1 \mathrm{H}, \mathrm{dd},{ }^{3} J_{\mathrm{HH}} 7.2 \mathrm{~Hz},{ }^{4} J_{\mathrm{HH}} 1.6 \mathrm{~Hz}, \mathrm{ArH}\right), 6.54\left(1 \mathrm{H}, \mathrm{q},{ }^{4} J_{\mathrm{HH}} 1.6 \mathrm{~Hz}, \mathrm{ArCH}\right), 3.37\left(2 \mathrm{H}, \mathrm{s}, \mathrm{ArCH}_{2}\right)$,

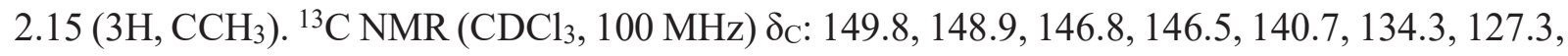
127.1, 123.6, 123.2, 120.1, 42.5, 16.6. EI-HRMS $(\mathrm{m} / z)$ calcd for $\mathrm{C}_{15} \mathrm{H}_{13} \mathrm{~N}\left(\mathrm{M}^{+}\right)$207.1048, found 207.1053 .

5-(2-Methyl-1H-inden-7-yl)pyrimidine (10 $\left.b_{3}\right)$. Yield 79\%, $164 \mathrm{mg}$, light yellow solid, Mp 70$72{ }^{\circ} \mathrm{C} .{ }^{1} \mathrm{H} \mathrm{NMR}\left(\mathrm{CDCl}_{3}, 400 \mathrm{MHz}\right) \delta_{\mathrm{H}}: 9.20(1 \mathrm{H}, \mathrm{s}, \mathrm{ArH}), 8.90(2 \mathrm{H}, \mathrm{s}, \mathrm{ArH}), 7.31-7.38(2 \mathrm{H}, \mathrm{m}$, ArH), $7.09\left(1 \mathrm{H}, \mathrm{dd},{ }^{3} J_{\mathrm{HH}} 7.2 \mathrm{~Hz},{ }^{4} J_{\mathrm{HH}} 0.8 \mathrm{~Hz}, \mathrm{ArH}\right), 6.55\left(1 \mathrm{H}, \mathrm{q},{ }^{4} J_{\mathrm{HH}} 1.6 \mathrm{~Hz}, \mathrm{ArCH}\right), 3.35(2 \mathrm{H}, \mathrm{s}$,

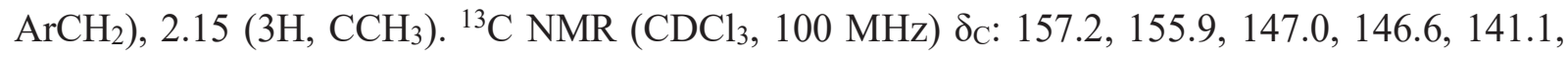
$134.7,129.9,127.6,127.1,123.8,120.4,42.3,16.6$. EI-HRMS $(m / z)$ calcd for $\mathrm{C}_{14} \mathrm{H}_{12} \mathrm{~N}_{2}\left(\mathrm{M}^{+}\right)$ 208.1000, found 208.1008.

3-(2-Methyl-1H-inden-7-yl)quinoline (10b4). Yield 53\%, $136 \mathrm{mg}$, white solid, Mp 190-192 ${ }^{\circ} \mathrm{C}$. ${ }^{1} \mathrm{H} \mathrm{NMR}\left(\mathrm{CDCl}_{3}, 400 \mathrm{MHz}\right) \delta_{\mathrm{H}} 9.12\left(1 \mathrm{H}, \mathrm{d},{ }^{4} J_{\mathrm{HH}} 2.0 \mathrm{~Hz}, \mathrm{ArH}\right), 8.24\left(1 \mathrm{H}, \mathrm{d},{ }^{4} J_{\mathrm{HH}} 2.0 \mathrm{~Hz}, \mathrm{ArH}\right)$, $8.16\left(1 \mathrm{H}, \mathrm{d},{ }^{3} J_{\mathrm{HH}} 8.4 \mathrm{~Hz}, \mathrm{ArH}\right), 7.85\left(1 \mathrm{H}, \mathrm{d},{ }^{3} J_{\mathrm{HH}} 8.4 \mathrm{~Hz}, \mathrm{ArH}\right), 7.73\left(1 \mathrm{H}, \mathrm{dt},{ }^{3} J_{\mathrm{HH}} 7.6 \mathrm{~Hz},{ }^{4} J_{\mathrm{HH}} 1.6\right.$ $\mathrm{Hz}, \mathrm{ArH}), 7.57\left(1 \mathrm{H}, \mathrm{dt},{ }^{3} J_{\mathrm{HH}} 7.6 \mathrm{~Hz},{ }^{4} J_{\mathrm{HH}} 1.2 \mathrm{~Hz}, \mathrm{ArH}\right), 7.37\left(1 \mathrm{H}, \mathrm{d},{ }^{3} J_{\mathrm{HH}} 7.6 \mathrm{~Hz}, \mathrm{ArH}\right), 7.33(1 \mathrm{H}$, dd, $\left.{ }^{3} J_{\mathrm{HH}} 7.2 \mathrm{~Hz},{ }^{4} J_{\mathrm{HH}} 0.8 \mathrm{~Hz}, \mathrm{ArH}\right), 7.22\left(1 \mathrm{H}, \mathrm{dd},{ }^{3} J_{\mathrm{HH}} 7.6 \mathrm{~Hz},{ }^{4} J_{\mathrm{HH}} 1.0 \mathrm{~Hz}, \operatorname{ArH}\right), 6.58(1 \mathrm{H}, \mathrm{q}$, 
$\left.{ }^{4} J_{\mathrm{HH}} 1.6 \mathrm{~Hz}, \mathrm{ArCH}\right), 3.41\left(2 \mathrm{H}, \mathrm{s}, \mathrm{ArCH}_{2}\right), 2.16\left(3 \mathrm{H}, \mathrm{CCH}_{3}\right) .{ }^{13} \mathrm{C} \mathrm{NMR}\left(\mathrm{CDCl}_{3}, 100 \mathrm{MHz}\right) \delta_{\mathrm{C}}$ : 150.8, 147.1, 146.8, 146.5, 141.3, 134.5, 134.1, 133.7, 129.3, 129.2, 127.9, 127.3, 127.1, 126.9, 124.4, 119.7, 42.6, 16.6. EI-HRMS $(\mathrm{m} / \mathrm{z})$ calcd for $\mathrm{C}_{19} \mathrm{H}_{15} \mathrm{~N}\left(\mathrm{M}^{+}\right) 257.1204$, found 257.1205.

2-(2-Methyl-1H-inden-7-yl)thiophene (10b5). Yield 77\%, $163 \mathrm{mg}$, brown oil. ${ }^{1} \mathrm{H} \mathrm{NMR}\left(\mathrm{CDCl}_{3}\right.$, $400 \mathrm{MHz}) \delta_{\mathrm{H}}: 7.33-7.39(2 \mathrm{H}, \mathrm{m}, \mathrm{ArH}), 7.29-7.32(1 \mathrm{H}, \mathrm{m}, \mathrm{ArH}), 7.26\left(1 \mathrm{H}, \mathrm{d},{ }^{3} J_{\mathrm{HH}} 7.6 \mathrm{~Hz}, \mathrm{ArH}\right)$, $7.19\left(1 \mathrm{H}, \mathrm{d},{ }^{3} \mathrm{~J}_{\mathrm{HH}} 8.0 \mathrm{~Hz}, \mathrm{ArH}\right), 7.08-7.12(1 \mathrm{H}, \mathrm{m}, \mathrm{ArH}), 6.57(1 \mathrm{H}, \mathrm{s}, \mathrm{ArCH}), 3.37\left(2 \mathrm{H}, \mathrm{s}, \mathrm{ArCH}_{2}\right)$, $2.17\left(3 \mathrm{H}, \mathrm{CCH}_{3}\right)$.

2-(2-Methyl-1H-inden-7-yl)furan (10b $)$ ). Yield 63\%, $123 \mathrm{mg}$, colorless oil. ${ }^{1} \mathrm{H} \mathrm{NMR}\left(\mathrm{CDCl}_{3}\right.$, $400 \mathrm{MHz}) \delta_{\mathrm{H}}: 7.50\left(1 \mathrm{H}, \mathrm{d},{ }^{3} J_{\mathrm{HH}} 7.8 \mathrm{~Hz}, \mathrm{ArH}\right), 7.47\left(1 \mathrm{H}, \mathrm{d},{ }^{4} J_{\mathrm{HH}} 1.3 \mathrm{~Hz}, \mathrm{ArH}\right), 7.25\left(1 \mathrm{H}, \mathrm{t},{ }^{3} J_{\mathrm{HH}}\right.$ $7.6 \mathrm{~Hz}, \mathrm{ArH}), 7.16\left(1 \mathrm{H}, \mathrm{d},{ }^{3} J_{\mathrm{HH}} 7.2 \mathrm{~Hz}, \mathrm{ArH}\right), 6.61\left(1 \mathrm{H}, \mathrm{d},{ }^{4} J_{\mathrm{HH}} 3.2 \mathrm{~Hz}, \mathrm{ArH}\right), 6.46-6.48(2 \mathrm{H}, \mathrm{m}$,

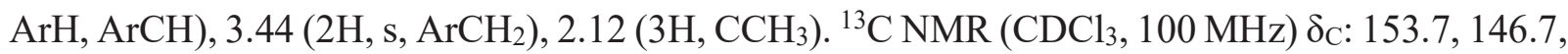
146.2, 141.6, 137.9, 126.8, 126.7, 125.9, 120.1, 119.0, 111.4, 106.7, 43.8, 16.7. EI-HRMS $(\mathrm{m} / \mathrm{z})$ calcd for $\mathrm{C}_{14} \mathrm{H}_{12} \mathrm{O}\left(\mathrm{M}^{+}\right) 196.0888$, found 196.0897

Multi-gram scale synthesis of $10 \mathrm{a}_{15}$ without fraction distillation or column chromatography Into a $100 \mathrm{~mL}$ round-bottom flask, was filled with a mixture of 4-bromo-2-methyl 1-indanone 7 (4.50 g, 20 mmol), 3,5-ditrifluoromethyl phenylboronic acid 8a $\mathbf{8 5}$ (5.40 g, $21 \mathrm{mmol}, 1.05$ eq.), $\mathrm{Pd}(\mathrm{OAc})_{2}$ (0.225 mg, $1.0 \mathrm{umol}, 0.005 \mathrm{~mol} \%$ ), TBAB (7.16 g, $20 \mathrm{mmol}, 1.0$ eq.), $\mathrm{K}_{2} \mathrm{CO}_{3}$ (5.53g, $40 \mathrm{mmol}, 2.0$ eq.) and PEG400 $50 \mathrm{~g}$. The mixture was stirred at $110^{\circ} \mathrm{C}$ till complition (TLC). 50 $\mathrm{mL}$ of water was added and the contents were extracted with EtOAc (100 mL X 3). The organic phase was washed with brine (100 mL X 2), dried over $\mathrm{Na}_{2} \mathrm{SO}_{4}$ and concentrated to dryness. 20 $\mathrm{mL}$ of methanol was added and the suspension was stirred overnight. After filtration, the white solid was dissolved in $300 \mathrm{~mL}$ of THF/MeOH (2:1) and $1.14 \mathrm{~g} \mathrm{(30} \mathrm{mmol,} 1.5$ eq.) of $\mathrm{NaBH}_{4}$ was added in portions at $0{ }^{\circ} \mathrm{C}$. The reaction was stirred at rt till completion (TLC). After evaporation, the residue was partitioned between EtOAc and $\mathrm{H}_{2} \mathrm{O}(200 \mathrm{~mL}, 1: 1)$. The aqueous phase was extracted with EtOAc $(100 \mathrm{~mL} \times 2)$. The combined organic extracts were dried and evaporated to dryness. The crude product was taken up in $350 \mathrm{~mL}$ of toluene and mixed with $200 \mathrm{mg}$ of TsOH monohydrate. The formed mixture was refluxed with Dean-Stark head for $5 \mathrm{~h}$. Toluene was removed under reduce pressure and the residue was re-dissolved in EtOAc/MeOH (200 mL, 1:1). The aqueous phase was extracted with EtOAc $(100 \mathrm{~mL} \times 2)$. The combined organic extracts were washed subsequently with saturated $\mathrm{NaHCO}_{3}(100 \mathrm{~mL} \times 2)$, brine $(100 \mathrm{~mL} \times 2)$ and dried over $\mathrm{Na}_{2} \mathrm{SO}_{4}$. After evaporation, the residue was suspended in methanol $(15 \mathrm{~mL})$ and stirred overnight. The slurry was filtered to yield 2-methyl-7-(3,5-bistrifluoromethylphenyl)indene 10a15 $5.9 \mathrm{~g}$ (17.2 $\mathrm{mmol}$ ) in $85.8 \%$ yield. 


\section{Acknowledgements}

The authors thank the Fundamental Research Funds for the Central Universities (No. DUT14LAB19) and CNPC (Project No. 2011B-2703-0104) for financial support. We also thank Prof. Baomin Wang, Prof. Yuhan Zhou, and Prof. Ying Peng for valuable discussions.

\section{References}

1. Spaleck, W.; Kueber, F.; Winter, A.; Rohrmann, J.; Bachmann, B.; Antberg, M.; Dolle, V.; Paulus, E. F. Organometallics 1994, 13, 954. http://dx.doi.org/10.1021/om00015a032

2. Togni, A.; Halterman, R. L. Metallocenes; Wiley-VCH, 1998.

3. Chisholm, M.; Zhou, Z.; Baugh, L.; Canich, J. Stereoselective Polymerization with Single Site Catalysts CRC Press Boca Raton, FL: 2008.

4. Wang, H.-Y.; Guo, C.-Y.; Dong, J.-Y. Cat. Commun. 2008, 10, 61. http://dx.doi.org/10.1016/j.catcom.2008.07.043

5. Ostoja Starzewski, A.; Steinhauser, N.; Xin, B. S. Macromolecules 2008, 41, 4095. http://dx.doi.org/10.1021/ma8007306

6. De Rosa, C.; Auriemma, F.; Di Girolamo, R.; Romano, L.; De Luca, M. R. Macromolecules 2010, 43, 8559. http://dx.doi.org/10.1021/ma101543d

7. Stagnaro, P.; Boragno, L.; Losio, S.; Canetti, M.; Alfonso, G. C.; Galimberti, M.; Piemontesi, F.; Sacchi, M. C. Macromolecules 2011, 44, 3712. http://dx.doi.org/10.1021/ma102914r

8. Ushakova, T.; Starchak, E.; Krasheninnikov, V.; Samoilenko, A.; Ivchenko, P.; Nifant'ev, I.; Novokshonova, L. Kinetics Catal. 2012, 53, 75. http://dx.doi.org/10.1134/S0023158412010156

9. Paavola, S.; Löfgren, B.; Seppälä, J. V. Eur. Polym. J. 2005, 41, 2861. http://dx.doi.org/10.1016/j.eurpolymj.2005.05.014

10. Lin, W.; Dong, J.; Chung, T. M. Macromolecules 2008, 41, 8452. http://dx.doi.org/10.1021/ma801469s

11. Atiqullah, M.; Tinkl, M.; Pfaendner, R.; Akhtar, M.; Hussain, I. Polymer Rev. 2010, 50, 178. http://dx.doi.org/10.1080/15583721003704289

12. Zhang, M.; Colby, R. H.; Milner, S. T.; Chung, T. M.; Huang, T.; deGroot, W. Macromolecules 2013, 46, 4313. http://dx.doi.org/10.1021/ma4006632

13. Zhang, M.; Yuan, X.; Wang, L.; Chung, T. M.; Huang, T.; deGroot, W. Macromolecules 2014, 47, 571. http://dx.doi.org/10.1021/ma402328k 
14. Zhang, G.; Li, H.; Antensteiner, M.; Chung, T. M. Macromolecules 2015, 48, 2925. http://dx.doi.org/10.1021/acs.macromol.5b00439

15. Brintzinger, H. H.; Fischer, D. In Polyolefins: 50 years after Ziegler and Natta II; Springer: 2013, p 29.

16. Nifant'ev, I. E.; Ivchenko, P. V.; Bagrov, V. V.; Okumura, Y.; Elder, M.; Churakov, A. V. Organometallics 2011, 30, 5744.

http://dx.doi.org/10.1021/om200610b

17. Ewen, J. A.; Elder, M. J.; Jones, R. L.; Rheingold, A. L.; Liable-Sands, L. M.; Sommer, R. D. J. Am. Chem. Soc. 2001, 123, 4763.

http://dx.doi.org/10.1021/ja004266h

18. Nifant'ev, I. E.; Ivchenko, P. V.; Bagrov, V. V.; Churakov, A. V.; Mercandelli, P. Organometallics 2012, 31, 4962.

http://dx.doi.org/10.1021/om300160v

19. Schaverien, C. J.; Ernst, R.; Schut, P.; Dall'Occo, T. Organometallics 2001, 20, 3436. http://dx.doi.org/10.1021/om010160z

20. Bingel, C.; Goeres, M.; Fraaije, V.; Winter, A.; WO9840331A1; 1998. Chem. Abstr. 1998, 129,260575

21. Ivchenko, P.; Nifant'ev, I. Russ. Chem. Bull. 2008, 57, 1661. http://dx.doi.org/10.1007/s11172-008-0217-2

22. Izmer, V. V.; Lebedev, A. Y.; Nikulin, M. V.; Ryabov, A. N.; Asachenko, A. F.; Lygin, A. V.; Sorokin, D. A.; Voskoboynikov, A. Z. Organometallics 2006, 25, 1217. http://dx.doi.org/10.1021/om050937e

23. Nikulin, M.; Tsarev, A.; Lygin, A.; Ryabov, A.; Beletskaya, I.; Voskoboinikov, A. Russ. Chem. Bull. 2008, 57, 2298. http://dx.doi.org/10.1007/s11172-008-0325-Z

24. Schöbel, A.; Herdtweck, E.; Parkinson, M.; Rieger, B. Chem. Eur. J. 2012, 18, 4174. http://dx.doi.org/10.1002/chem.201103547

25. Ryabov, A. N.; Izmer, V. V.; Tzarev, A. A.; Uborsky, D. V.; Asachenko, A. F.; Nikulin, M. V.; Canich, J. A. M.; Voskoboynikov, A. Z. Organometallics 2009, 28, 3614. http://dx.doi.org/10.1021/om900353m

26. Greger, I.; Kehr, G.; Fröhlich, R.; Erker, G. Organometallics 2010, $29,860$. http://dx.doi.org/10.1021/om900939v

27. Pinkas, J.; Lamač, M. Coordin. Chem. Rev. 2015, 296, 45. http://dx.doi.org/10.1016/j.ccr.2015.03.007

28. Laine, A.; Linnolahti, M.; Pakkanen, T. A.; Severn, J. R.; Kokko, E.; Pakkanen, A. Organometallics 2011, 30, 1350. http://dx.doi.org/10.1021/om100761p

29. Talarico, G.; Budzelaar, P. H. Organometallics 2014, 33, 5974. http://dx.doi.org/10.1021/om5003655 
30. Kuklin, M. S.; Virkkunen, V.; Castro, P. M.; Resconi, L.; Linnolahti, M. Eur. J. Inorg. Chem. 2015, 4420.

http://dx.doi.org/10.1002/ejic.201500862

31. Laine, A.; Coussens, B. B.; Hirvi, J. T.; Berthoud, A.; Friederichs, N.; Severn, J. R.; Linnolahti, M. Organometallics 2015, 34, 2415.

http://dx.doi.org/10.1021/om501185x

32. Miyaura, N.; Suzuki, A. Chem. Rev. 1995, 95, 2457.

http://dx.doi.10.1021/cr00039a007

33. Kotha, S.; Lahiri, K.; Kashinath, D. Tetrahedron 2002, 58, 9633.

http://dx.doi:10.1016/S0040-4020(02)01188-2

34. Bellina, F.; Carpita, A.; Rossi, R. Synthesis 2004, 2419.

http://dx.doi: 10.1055/s-2004-831223

35. Kotha, S.; Lahiri, K. Eur. J. Org. Chem. 2007, 1221.

http://dx.doi.org/10.1002/ejoc.200600519

36. Liu, L.; Zhang, Y.; Wang, Y. J. Org. Chem. 2005, 70, 6122.

http://dx.doi.org/10.1021/ jo050724z

37. Liu, W.-J.; Xie, Y.-X.; Liang, Y.; Li, J.-H. Synthesis. 2006, 860.

http://dx.doi.org/10.1055/s-2006-926323

38. Han, W.; Liu, C.; Jin, Z.-L. Org. Lett. 2007, 9, 4005.

http://dx.doi.org/10.1021/o1701709q

39. Razler, T. M.; Hsiao, Y.; Qian, F.; Fu, R.; Khan, R. K.; Doubleday, W. J. Org. Chem. 2008, $74,1381$.

http://dx.doi.org/10.1021/ jo802277z 